Draft Version OCtober 13, 2020

Typeset using LATEX twocolumn style in AASTeX61

\title{
NUMERICAL SIMULATIONS OF THE JET DYNAMICS AND SYNCHROTRON RADIATION OF BINARY NEUTRON STAR MERGER EVENT GW170817/GRB170817A
}

\author{
Xiaoyi Xie, ${ }^{1}$ Jonathan Zrake, ${ }^{2}$ And Andrew MacFadyen ${ }^{1}$ \\ ${ }^{1}$ Center for Cosmology and Particle Physics, Physics Department New York University, 726 Broadway, New York, NY 10003, USA \\ ${ }^{2}$ Columbia University, Pupin Hall, 550 West 120th Street, New York, NY 10027, USA
}

\section{Submitted to ApJ}

\begin{abstract}
We present numerical simulations of relativistic outflows propagating through the debris cloud of a binary neutron star (BNS) merger. Starting from the scale of the central engine, we use a moving-mesh hydrodynamics code to simulate powerful relativistic outflows produced in BNS merger environments. We compute synchrotron emission directly from the simulations and present multi-band light curves of the early (sub-day) through late (weeks to years) afterglow stages. Our work systematically compares two distinct models for the central engine, referred to as the narrow and wide engine scenarios, which are associated with a successful structured jet and a quasi-isotropic explosion respectively. Both models naturally evolve angular and radial structure through hydrodynamical interaction with the merger debris cloud. They both also result in a relativistic blast wave capable of producing the observed multi-band data. However, we find that the narrow and wide engine scenarios might be differentiated by a new emission component that we refer to as a merger flash. Its rapidly declining signature may be detectable for future BNS mergers during the first minutes to day following the GW chirp. Furthermore, its non-detection for the GRB170817 event may disfavor the wide, quasi-isotropic explosion model.
\end{abstract}

Keywords: hydrodynamics — shock waves - radiation mechanisms: non-thermal — gamma-ray burst: individual (GRB170817) — stars: neutron 


\section{INTRODUCTION}

On August 17, 2017 the Laser Interferometer Gravitational Wave Observatory (LIGO) detected the first gravitational wave (GW) signal from the merger of a binary neutron star system (Abbott et al. 2017). About 1.7 s later, the Fermi Gamma-ray Burst Monitor (GBM) detected a coincident short Gamma-Ray Burst (sGRB), marking the first confident joint electromagnetic (EM)-gravitational wave (GW) observation in history (Goldstein et al. 2017; Savchenko et al. 2017). Follow-up observing campaigns across the electromagnetic spectrum were launched to discover the merger site and observe its ongoing electromagnetic emission. In less than 11 hours after the merger, a bright optical transient was discovered in the galaxy NGC4993 (at $40 \mathrm{Mpc}$ ) (Coulter et al. 2017; Abbott et al. 2017; Soares-Santos et al. 2017; Valenti et al. 2017). Early ultraviolet-optical-infrared (UVOIR) data from multiple telescopes throughout the world reveal a quasi-thermal radiation component, which is consistent with the prediction of the "kilonova/macronova" model (Metzger 2017; Chornock et al. 2017; Cowperthwaite et al. 2017; Nicholl et al. 2017; Smartt et al. 2017; Soares-Santos et al. 2017; Tanvir et al. 2017; Villar et al. 2017; Kasliwal et al. 2017; Kasen et al. 2017; Pian et al. 2017). However, no X-ray and radio signals were detected during the first several days, though important flux upperlimits were obtained (Abbott et al. 2017). The first detection of X-rays came from Chandra 9 days after the GW event (Margutti et al. 2017; Troja et al. 2017). Radio emission was first detected 16 days after the GW event (Alexander et al. 2017).

Continuous X-ray and radio observations show steadily increasing luminosity up to $\sim 100$ days after the GW event (Haggard et al. 2017; Hallinan et al. 2017; Mooley et al. 2018; Ruan et al. 2018). Recent observations ( $\sim 200$ days after GW) may show a hint of a turn-over in the radio, optical and X-ray light curves (Lyman et al. 2018; Margutti et al. 2018; D'Avanzo et al. 2018; Dobie et al. 2018).

The late-time non-thermal light curves can be interpreted as synchrotron emission from a relativistic blast wave launched from the merger and propagating in the circum-merger environment (hereafter, referred to as the interstellar medium (ISM)). Several scenarios have been proposed for launching the blast wave during the merger process. On-axis and off-axis "top-hat" Blandford-McKee (BM) (Blandford \& McKee 1976) jet models, for which the energy and radial velocity are uniform within a cone and drop discontinuously to zero outside of the cone, have been ruled out (see Mooley et al. 2018; Kasliwal et al. 2017). Two dynamical models for the central engine remain under consideration: 1) a narrow ultra-relativistic engine that produces a successful jet and 2) a wide trans-relativistic engine that does not. The first scenario has been referred to as a "successful structured jet" (e.g., Margutti et al. 2018), and the second has been referred to variously as a "choked jet" or "failed jet" (Kasliwal et al. 2017; Gottlieb et al. 2017; Troja et al. 2017; Mooley et al. 2018; Nakar et al. 2018).

In this work, we utilize the moving-mesh relativistic hydrodynamics code JET (Duffell \& MacFadyen 2013) coupled to a well-tested synchrotron radiation code (Zhang \& MacFadyen 2009; van Eerten et al. 2010) to conduct high-resolution full-time-domain simulations of these two dynamical models, starting at the scale of the central engine and evolving continuously to the scale of the afterglow. Broad-band light curves computed from these simulations can be used to interpret data from future BNS mergers expected to occur at rates of up to $1 /$ month after advanced LIGO and Virgo commence operation in Dec, 2018. We find that both dynamical models are consistent with current multi-frequency afterglow observations of the GW170817/GRB170817A event. However, our simulations reveal the possibility of an early rapidly-declining synchrotron emission component, which we refer to as a "merger flash." Unlike late afterglow emission, we suggest that rapid follow-up Xray observations (minutes to hours after the GW event) of the merger flash (including its non-detection) may aid in distinguishing between models.

The details of the numerical setup for both models are presented in Section 2. In Section 3, we demonstrate that successful jets that propagate through, and break out of the NS merger debris cloud naturally develop an angular structure through interaction with the merger debris. We discuss and analyze the dynamics and late afterglow radiation of successful structured jets and present off-axis afterglow light curves that match current observations of the GRB170817A afterglow. In Section 4 , we present simulations of the wide engine model and analyze its dynamics and radiative signatures. We then draw comparisons between the narrow engine model and the wide engine model. In Section 5 we discuss the multiple stages in the computed X-ray light curve. In Section 6 , we introduce the "merger flash", an early rapidly declining light curve component lasting minutes to hours following the GW signal, that might be detectable by current or proposed observatories in the minutes following future BNS mergers. A soft X-ray merger flash following GW170817 may have been missed by Swift XRT, due to Earth occultation. For future nearby BNS mergers, the follow-up detection of the merger flash may be possible, particularly at X-ray energies, and may help 
constrain the observer viewing angle. We conclude in Section 7 with a summary of our findings.

\section{NUMERICAL SETUP}

\subsection{Initial conditions}

Our numerical setup captures the features of BNS merger environments that shape the dynamics of the relativistic outflow and its radiative signature. General relativistic magnetohydrodynamics (GRMHD) simulations of BNS mergers indicate that between $10^{-4}$ and $10^{-2}$ solar masses of neutron star material are ejected during the coalescence, forming a quasi-spherical debris cloud. The cloud expands mildly relativistically, with typical radial velocity $\sim 0.15-0.25$ c (e.g. Hotokezaka et al. 2013; Shibata et al. 2017). The modeling of the "kilonova" emission associated with GW170817 reveals that $\sim 10^{-2} \mathrm{M}_{\odot}$ of neutron rich material was ejected during the coalescence. We use this cloud mass in our simulations. The ejecta cloud has a slightly oblate geometry and radial stratification; most of its mass is confined in a slow-moving core, while a small amount of mass $10^{-4} \mathrm{M}_{\odot}$ lies in an extended fast-moving tail,

$$
\begin{aligned}
\rho_{c}(r, \theta) & =\rho_{c}\left(r / r_{c}\right)^{-2}\left(1 / 4+\sin ^{3} \theta\right) \quad r<r_{c}, \\
\rho_{t}(r) & =\rho_{t}\left(r / r_{c}\right)^{-n} \quad r_{c}<r<4 r_{c}, \\
v(r) & =v_{c} r / r_{c} \quad r<4 r_{c} .
\end{aligned}
$$

The density values $\rho_{c}$ and $\rho_{t}$ are calculated based on the total mass of the slow core and fast tail. The density power-law index $n$ is set to $8 . v_{c}=0.2 \mathrm{c}$ sets the maximal velocity of the core. $r_{c}=1.3 \times 10^{9} \mathrm{~cm}$ is the core radius. This initial condition is similar to Kasliwal et al. 2017; Gottlieb et al. 2017. Both the narrow engine model and the wide engine model are evolved in the same merger cloud.

The central engine is initiated when the cloud has evolved for 1 second after the BNS coalescence. We make this choice because it yields GRB prompt emission compatible with the $1.7 \mathrm{~s}$ time delay between the observation of the GW chirp and the sGRB signal (Kasliwal et al. 2017; Gottlieb et al. 2017; Nakar et al. 2018). The total engine energy is fixed at $5 \times 10^{50} \mathrm{erg}$ (per hemisphere). The engine total energy corresponds to roughly $6 \%$ of the rest mass energy of the merger. Our simulation parameters are summarized in Table 1.

\subsection{Numerical methods}

We conduct 2D axially symmetric relativistic hydrodynamic simulations with the moving mesh code - JET (Duffell \& MacFadyen 2013). The jet engine is injected as a source term for both models. For the narrow engine model, we choose the jet engine as a nozzle with circular profile (Duffell et al. 2015). For the wide engine model, we adopt the same injection method of Kasliwal et al. 2017; Gottlieb et al. 2017, where a cylindrical nozzle is used. Throughout, we use an ideal gas equation of state with adiabatic index $4 / 3$.

Our simulations take place on a spherical grid with $N_{\theta}=160$ zones, evenly distributed in polar angle over the half-sphere. The central engine is modeled by injecting relativistic flow near the cloud center. The radial grid is logarithmically spaced so that the cell aspect ratio is close to one. Each simulation cell face moves radially with the flow. We adopt an adaptive mesh refinement (AMR) scheme that dynamically refines regions with high Lorentz factor.

Table 1. Hydro parameters for the narrow engine model and the wide engine model.

\begin{tabular}{lcc}
\hline \hline \multicolumn{1}{c}{ Variable } & Narrow Engine & Wide Engine \\
\hline$M_{\text {cloud }}(D S)$ & $0.01 \mathrm{M}_{\odot}$ & $0.01 \mathrm{M}_{\odot}$ \\
$L_{\text {jet }}(S S)$ & $2.6 \times 10^{50} \mathrm{erg} \mathrm{s}^{-1}$ & $2.6 \times 10^{50} \mathrm{erg} \mathrm{s}^{-1}$ \\
$t_{\text {jet }}$ & $2 \mathrm{~s}$ & $2 \mathrm{~s}$ \\
$\Gamma_{0}$ & 10 & 1.02 \\
$\eta$ & 100 & 20 \\
$\theta_{\text {jet }}$ & 0.1 & 0.35 \\
$n_{\text {ism }}$ & $10^{-4}, 10^{-5} \mathrm{~cm}^{-3}$ & $10^{-5} \mathrm{~cm}^{-3}$ \\
\hline
\end{tabular}

Note- $D S(S S)$ represents double-sided (single-sided). We use the same merger cloud, the same jet engine luminosity $L_{\text {jet }}$ and engine duration $t_{\text {jet }}$ for both the narrow engine model and the wide engine model. The cloud mass $M_{\text {cloud }}$ is $\sim 0.01 \mathrm{M}_{\odot}$. The initial Lorentz factor $\Gamma_{0}$, the specific enthalpy $\eta$, and the half opening angle $\theta_{\text {jet }}$ of the jet engine are set to different values between these two models.

\section{SUCCESSFUL JETS AND DEVELOPMENT OF ANGULAR STRUCTURE}

Here we report the dynamics and afterglow signature of a simulation model in which the central engine produces a successful relativistic jet, that is, one that successfully breaks out of the merger cloud and continues propagating into the ISM.

\subsection{Development of the angular structure}

In modeling a successful jet, we inject hot, relativistic material within a narrow opening angle (see Table 1). The jet drills through the dense core of the merger cloud, 


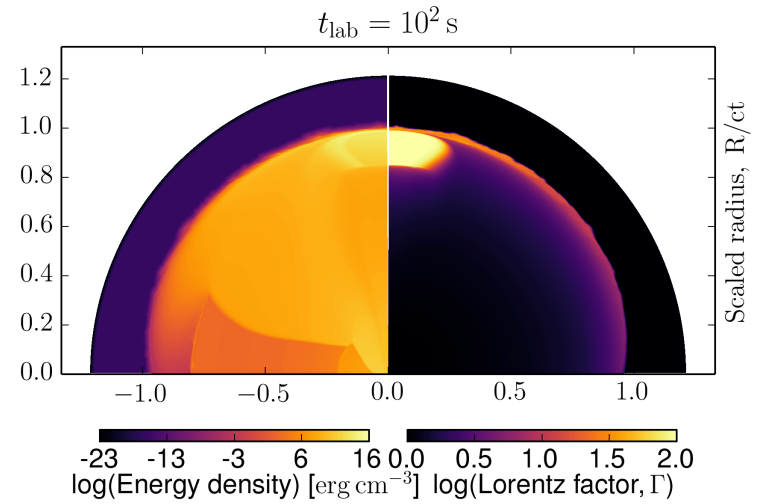

(a)

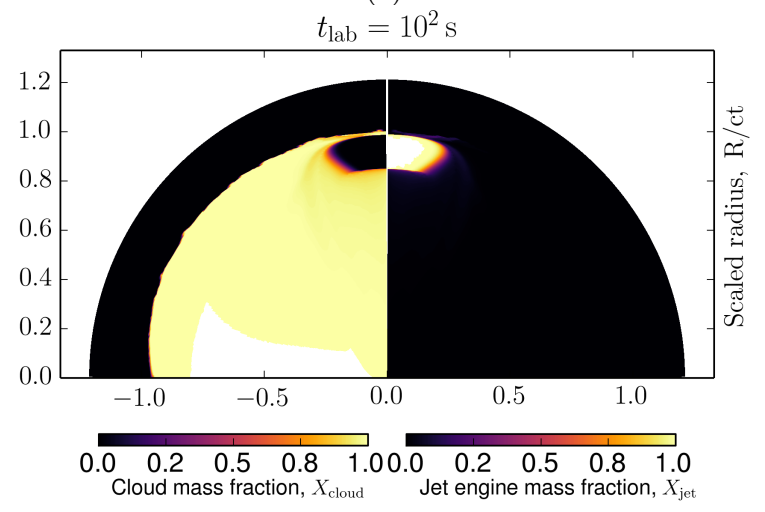

(b)

Figure 1. Narrow engine model - (a) The logarithmic total energy density (excluding rest mass; left panel) and the Lorentz factor $(\log \Gamma$, right panel) contour plot taken at the central lab time $t_{\text {lab }}=100 \mathrm{~s}$. (b) The mass fraction contour plot for the merger cloud $X_{\text {cloud }}$ (left panel) and the jet engine material $X_{\text {jet }}$ (right panel) taken at time $t_{\text {lab }}=100 \mathrm{~s}$. The early domain profile of the narrow engine model contains a still forming ultra-relativistic core, primarily composed of jet engine materials, surrounded by a mildly relativistic cocoon, composed of merger cloud materials.

and breaks out highly over-pressurized, driving sideways expansion in the fast-moving, lower density tail of the merger cloud. Eventually, the outflow escapes the cloud altogether, at a radius $\sim 2.4 \times 10^{11} \mathrm{~cm}$. GRB prompt emission photons are released from the vicinity of this break out radius (Kasliwal et al. 2017; Gottlieb et al. 2017; Nakar et al. 2018). Along the propagation direction, the relativistic GRB ejecta compresses a slowermoving sheath of merger debris ahead of it. This internal collision heats and compresses the sheath into a very thin ultra-relativistic shell. Meanwhile the rapid lateral expansion of the sideways shock accelerates a mildly relativistic cocoon of shocked neutron star material, extending to a large lateral angle, as shown in Figure 1.

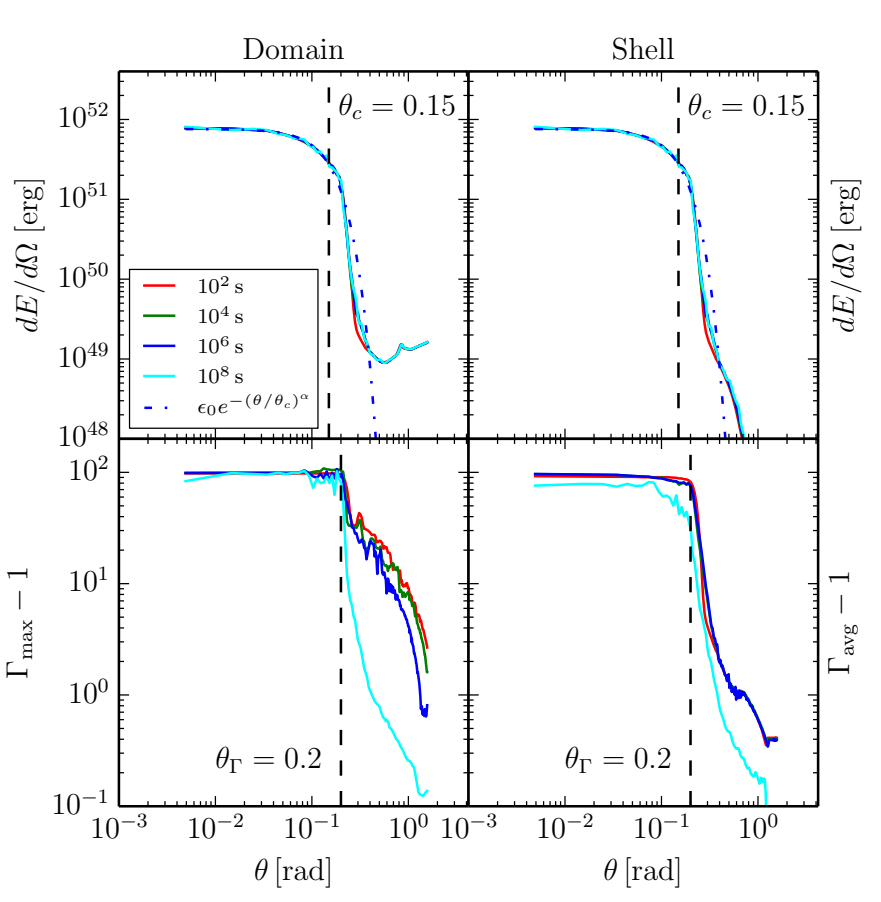

Figure 2. Narrow engine model - Shown in the top row is the angular distribution of the total energy (kinetic plus thermal). The bottom row shows the maximum Lorentz factor over the entire simulation domain (left panel) and the energyaveraged Lorentz factor for the relativistic shell (right panel). The columns correspond to data taken from the entire domain (left column) and from the relativistic shell (right column). Results from different time snapshots are colored with $t_{\text {lab }}=10^{2} \mathrm{~s}$ (red), $10^{4} \mathrm{~s}$ (green), $10^{6} \mathrm{~s}$ (blue), and $10^{8} \mathrm{~s}$ (cyan). The angular distribution of the total energy is well fitted by a quasi-Gaussian model $\epsilon_{0} e^{-\left(\theta / \theta_{c}\right)^{\alpha}}$ (dot-dashed line) with $4 \pi \epsilon_{0}=9.6 \times 10^{52} \mathrm{erg}, \theta_{c}=0.15$, and $\alpha=1.93$. The same fitting values are found to be appropriate for both the entire domain, and the volume occupied by the relativistic shell. The half opening angle $\theta_{c}=0.15$ is shown in vertical dashed, purple line. Angles to the left of the vertical line at $\theta_{\Gamma}=0.2$ lie in the relativistic core of the jet.

After having emerged from the cloud, the jet has developed an angular dependent structure. The angular distribution of the total energy (kinetic plus thermal) is shown qualitatively in Figure 1 and quantitatively in Figure 2. The jet contains an ultra-relativistic core $(\Gamma \sim 100)$, and a mildly relativistic sheath $(\Gamma \sim 10)$. In Figure 2, we differentiate between the relativistic shell $(\Gamma>1.1)$ and the entire domain, labeled as shell and domain, respectively.

We find that the angular energy distribution $(d E / d \Omega)$ for both components is well described by the quasiGaussian profile,

$$
d E / d \Omega=\epsilon_{0} e^{-\left(\theta / \theta_{c}\right)^{\alpha}}
$$


This angular energy distribution is different from the top-hat model typically used in the fitting of GRB afterglow light curves (e.g., van Eerten et al. 2012); it better resembles the model described in Zhang et al. (2004). The total energy and the energy-averaged Lorentz factor,

$$
\Gamma_{\mathrm{avg}} \equiv \frac{\int \Gamma E d V}{\int E d V}
$$

of the relativistic shell maintain their initial angular structure for a long period of time $\sim 10^{8} \mathrm{~s}$. In Equation $2, E$ is the local energy density (measured in the lab frame) and $\Gamma$ is the Lorentz factor of the fluid element. The maximum isotropic equivalent energy of the angular structured jet is $E_{\text {iso,peak }}=4 \pi \epsilon_{0} \approx 10^{53} \mathrm{erg}$. Within an opening angle $\theta_{c}=0.15$, the average isotropic equivalent energy of the relativistic core is $E_{\text {iso,avg }}=$ $6 \times 10^{52} \mathrm{erg}$, larger than the average isotropic equivalent energy $E_{\mathrm{k} \text {,iso }} \approx(1-3) \times 10^{51} \mathrm{erg}$, inferred for typical short GRBs, but still within the observed range (Fong et al. 2015).

The angular structure develops as a result of overpressurized relativistic ejecta escaping the merger cloud into the relatively dilute ambient medium. This results in significant lateral expansion (depicted in Figure 1), in addition to radial acceleration. Just prior to escaping from the merger cloud at a distance of $\sim 2.4 \times 10^{11} \mathrm{~cm}$, the relativistic ejecta is decelerated as it passes through a reconfinement shock, resulting from the inertia of the cloud. As a result of the shock, the relativistic ejecta's breakout Lorentz factor is decreased, and its pressure is increased. The jet propagating into the ambient medium now consists of a shock-heated, baryon-clean core, surrounded by a shock-heated sheath of NS merger ejecta material.

\subsection{Successful structured jet dynamical evolution}

After the jet is launched by the central engine, it accelerates by converting its internal energy into kinetic energy. Over the course of $10 \mathrm{~s}$ (as measured in the lab frame) the jet attains its terminal Lorentz factor $\eta=100$ (which is also the specific internal enthalpy of the engine material at the jet base). During its propagation through the ejecta cloud, the jet performs work on it. In order to determine how the energy is partitioned during this phase of the evolution, we have computed the thermal energy $E_{t}$ and the kinetic energy $E_{k}$ for each of three components - the jet material, the shocked merger cloud (sometimes referred to as "cocoon" mate-

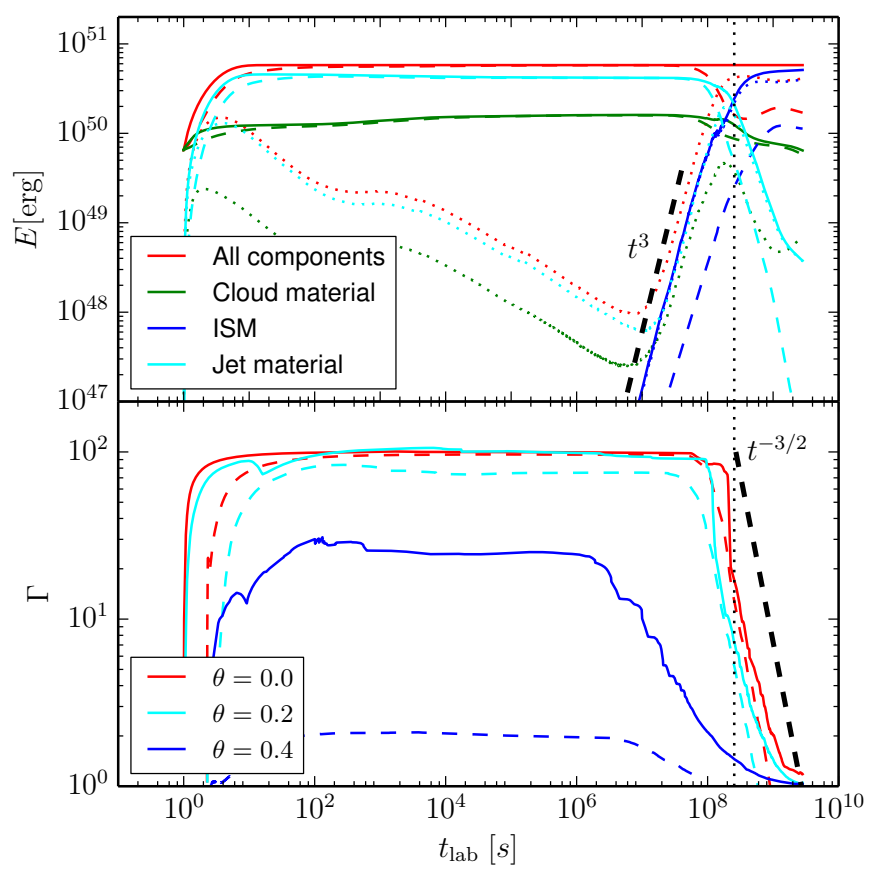

Figure 3. Narrow engine model - The dynamical evolution of various energy components (top panel) and the Lorentz factor (bottom panel), measured in the central lab frame. In the top panel, solid lines represent the total energy (kinetic plus thermal) for four components: everything in the domain (red), the ejecta cloud (green), the ISM (blue), and the jet engine (cyan). Dashed lines (dotted lines) represent kinetic energy (thermal energy) for each component. In less than 100 s, most of the thermal energy converts into the kinetic energy of the cloud and the jet. The residual thermal energy from these two components decreases afterwards in the process of adiabatic cooling. Meanwhile, the energy of the ISM steadily increases. At a later time $t_{\text {lab }}>10^{7} \mathrm{~s}$, the strong interaction among the jet, the cloud and the accumulated ISM efficiently converts kinetic energy back into thermal energy. The thermal energy of every component increases. In the bottom panel, the time evolution of the maximum Lorentz factor of the relativistic shell along different polar angles are shown in solid lines. The corresponding energy-averaged Lorentz factor are shown in dashed lines. Three dynamical stages are present in the entire lifecycle of the relativistic shell: rapid acceleration, the coasting, and the late deceleration.

rial), and the ISM. $E_{t}$ and $E_{k}$ are given by

$$
\begin{aligned}
& E_{t, i}=\int\left[(e+p) \Gamma^{2}-p\right] s_{i} d V \\
& E_{k, i}=\int[\Gamma(\Gamma-1) \rho h] s_{i} d V
\end{aligned}
$$

where $\rho, p, e$, and $h$ are the co-moving mass density, pressure, internal energy density, and specific gas enthalpy respectively, and $\Gamma$ is the Lorentz factor of the fluid. The subscript $i$ labels the individual components, 


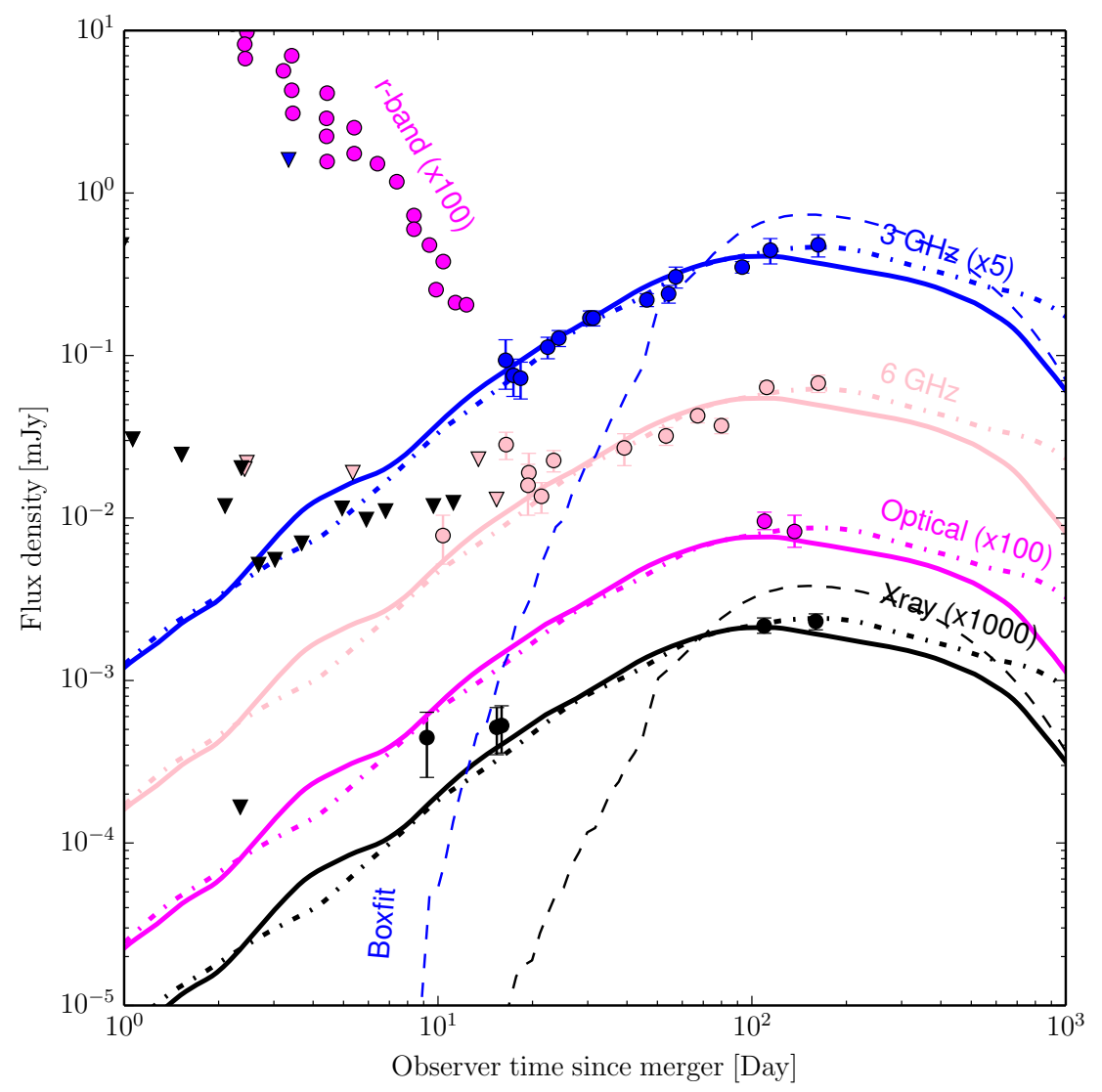

Figure 4. Narrow engine model — Two sets of fitting light curves calculated from numerical simulations of the successful structured jet propagating in two uniform ISM environments. The best-fit radiation parameter values, for the simulation with ISM density $n=10^{-4} \mathrm{~cm}^{-3}$, are $\theta_{\mathrm{obs}}=0.34\left(19.5^{\circ}\right), \epsilon_{e}=0.02, \epsilon_{B}=10^{-3}$, and $p=2.16$ (solid line). The best-fit radiation parameters for the simulation with ISM density $n=10^{-5} \mathrm{~cm}^{-3}$ are $\theta_{\text {obs }}=0.3\left(17^{\circ}\right), \epsilon_{e}=0.1, \epsilon_{B}=5 \times 10^{-4}$, and $p=2.16$ (dot-dashed line). These two sets of fitting light curves, smoothed by Savitzky-Golay filter, are consistent with both the early flux upper-limits for non-detection (down triangles) and the detected signals (dots with error bar). The non-detection upperlimits in Radio, X-ray and the non-thermal observations in Radio $(3 \mathrm{GHz}, 6 \mathrm{GHz})$, optical $\left(5 \times 10^{14} \mathrm{~Hz}\right)$, and X-ray $(1 \mathrm{keV})$ are all taken from Margutti et al. (2018). The early "kilonova" r-band data taken from Lyman et al. (2018) are also presented for comparison. The peak of the light curve is expected to come from the deceleration of the ultra-relativistic core of the successful structured jet. We make a comparison with the results from the top-hat jet model using BOXFIT (van Eerten et al. 2012). The isotropic equivalent energy and jet half opening angle for the top-hat jet model are set to the representative values of the successful structured jet: $E_{\text {iso, } 52}=6$ and $\theta_{j}=0.15$. With the same radiation parameter value $\theta_{\text {obs }}=0.34, \epsilon_{e}=0.02, \epsilon_{B}=10^{-3}$, $p=2.16$, and ISM density $n=10^{-4} \mathrm{~cm}^{-3}$, the light curve calculated from BOXFIT (dashed line) rises up faster compared with the light curve from the structured jet model. The peak time and the peak flux of the light curve between these two models are almost the same.

and the scalar field $s_{i}$ represents the fraction of each component filling the local volume $d V$; within each cell $\sum_{i=1}^{3} s_{i}=1$. This decomposition is accomplished by assigning to individual computational cells three passive scalar fields corresponding to the material components. The jet material is injected with $s=(1,0,0)$, the merger cloud material initially has $s=(0,1,0)$, and the ISM has $s=(0,0,1)$. As the simulation evolves, individual cells generally acquire some of each component due to mixing at the grid scale. To obtain $E_{k, i}$ and $E_{t, i}$ for each component $i$, we integrate Equations 3 over the volume.
The top panel of Figure 3 displays the time evolution of the kinetic and thermal energies in these three components. At the very beginning of jet propagation, the kinetic energy of the jet increases as it accelerates by expending its thermal energy supply. We also observe that simultaneously, the thermal energy content of the cloud material increases. This is the result of $P d V$ work, as well as shock heating, done by the jet on the cloud material as it drills through. After $\sim 100 \mathrm{~s}$, the jet reaches the outskirts of the merger cloud (see Figure 1). At this time its kinetic energy saturates and it stops performing 
work on the cloud material. The jet continues to cool adiabatically (see the dotted lines showing thermal energy in Figure 3) as it propagates into the circumburst environment.

The BNS merger event responsible for GW170817 occurred in the outskirts of an elliptical galaxy (Levan et al. 2017). Low ISM densities are not unusual in such environments, and therefore we have adopted values in the range $n=10^{-4}-10^{-5} \mathrm{~cm}^{-3}$ for the circumburst density, assumed to be constant for this discussion. In the co-moving frame of the relativistic shell, the upstream ISM particles stream inward with Lorentz factor $\Gamma$. When an ISM particle crosses the shock front, the direction of its velocity becomes random after multiple collisions. In the lab frame, the average energy of each downstream ISM proton is $\Gamma^{2} m_{p} c^{2}$. Detailed studies of jet dynamics and radiation have been covered in GRB reviews (e.g. Piran 1999; Mészáros 2006; Nakar 2007; Berger 2014; Kumar \& Zhang 2015). During the coasting phase of the relativistic jet, its bulk Lorentz factor does not change substantially. However, it performs work on the ISM, while at the same time accumulating mass. The total energy of the swept up ISM is given by

$$
E_{\text {iso, ism }} \approx \frac{4 \pi}{3} n R^{3} \Gamma^{2} \propto t^{3} .
$$

In Figure 3, the energy of the ISM is shown to be increasing throughout the coasting phase $\propto t^{3}$, in agreement with Equation $4 . E_{\text {iso,ism }}$ becomes comparable to the energy of the jet at lab time $t_{\text {lab }} \sim 2.5 \times 10^{8} \mathrm{~s}$. This is $\sim 3$ times longer than the predicted deceleration time $R_{d} / \mathrm{c}$, according to the estimate of Kumar \& Zhang (2015),

$$
\begin{aligned}
R_{d} & =\left(3 E_{\mathrm{iso}} / 4 \pi n m_{p} c^{2} \Gamma^{2}\right)^{1 / 3} \\
& \approx 10^{17} E_{\mathrm{iso}, 53}^{1 / 3} n^{-1 / 3} \Gamma_{2}^{-2 / 3} \mathrm{~cm},
\end{aligned}
$$

which yields a deceleration time of $7 \times 10^{7}$ s for our parameters.

The jet's transition from the coasting phase to the deceleration phase is accompanied by the formation of a strong forward shock, which then propagates into the ISM. A weaker reverse shock, which propagates into the jet ejecta also forms. Throughout the deceleration phase, the bulk Lorentz factor decays as $\Gamma \propto R^{-3 / 2} \propto$ $t^{-3 / 2}$ (Blandford \& McKee 1976; Kobayashi et al. 1999). In the bottom panel of Figure 3, the on-axis energyaveraged Lorentz factor $\Gamma_{\text {avg }}$ is shown to decay roughly as $\propto t^{-3 / 2}$ in agreement with the analytical estimate.

\subsection{Successful structured jet afterglow light curve}

Synchrotron emission using the model of Sari et al. (1998) can be calculated directly from multi-dimensional hydrodynamical simulation data in order to produce theoretical GRB afterglow light curves as a function of observer angle $\theta_{\text {obs }}$ with respect to the jet axis (e.g. van Eerten et al. 2010; De Colle et al. 2012). The main parameters determining the synchrotron radiation from the forward shock are the fraction $\epsilon_{B}$ of post-shock energy residing in magnetic fields, and the fraction $\epsilon_{e}$ in non-thermal electrons. We further adopt the convention that $\xi_{e}$ is the fraction of the electrons sharing the internal energy $\epsilon_{e} e$, and that the energy distribution of the relativistic non-thermal electrons is given by $d N / d \gamma \propto \gamma^{-p}$. We assume that $\xi_{e}=1$, and the electron spectral index $p$ is taken as a free parameter. We perform simulations of the successful structured jet propagating in low-density environments with two different values for the ISM density, $n=10^{-4}, 10^{-5} \mathrm{~cm}^{-2}$. By varying the value of the observer viewing angle $\theta_{\text {obs }}$ and the microphysical parameters $\left(\epsilon_{e}, \epsilon_{B}, p\right)$, we obtain two sets of off-axis light curves that match the broadband afterglow observations of GRB170817A.

The results of these fits are shown in Figure 4 (see also Margutti et al. 2018). Here we present light curves calculated from the structured jet simulation, contrasted with semi-analytical light curves computed using BOXFIT (van Eerten et al. 2012), and a simpler top-hat jet profile. The top-hat profile has the same isotropic equivalent energy, $E_{\text {iso,avg }}=6 \times 10^{52} \mathrm{erg}$, as the selfconsistently simulated jet, and we adopt an opening angle of $\theta_{c}=0.15$ taken from modeling the simulated jet according to Equation 1. Given the same radiation parameters, the light curves calculated from each model peak at roughly the same time, and exhibit similar peak fluxes. However, the early part of the afterglow light curve differs significantly between these two models. In particular, the off-axis light curve from the structured jet brightens earlier than the top-hat jet. The slope of the late decaying light curve from these two models is similar.

The late appearance of the X-ray and radio emission completely rules out any on-axis ultra-relativistic jet models. Indeed, if a relativistic top-hat jet had been pointed away from us, the afterglow emission would have been first detected at a later time, when the emission from the decelerated jet entered our line of sight. The rising light curve from the structured jet is robustly shallower than that of off-axis top-hat models (Mooley et al. 2018), and is thus detectable at much earlier times. The off-axis light curves from a structured jet naturally explain the GRB170817 afterglow emission.

\section{WIDE ENGINE MODEL}

In this section we explore the possibility that the afterglow of GRB170817 was the result of a wide central 


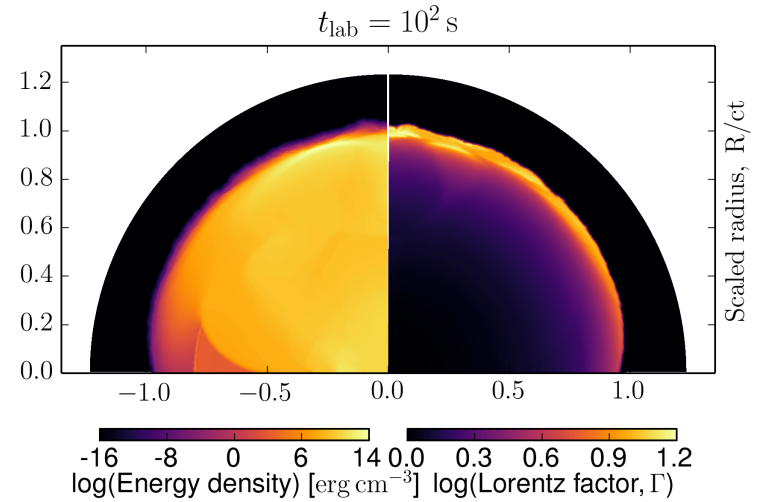

(a)

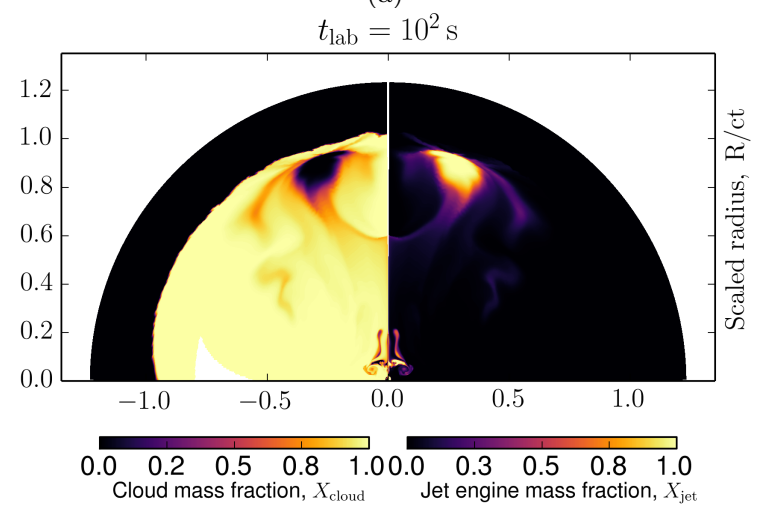

(b)

Figure 5. Wide engine model — Same as Figure 1, but for the wide engine model. The jet engine gets choked by the ejecta cloud, driving a wide spread mildly relativistic shell primarily composed of ejecta cloud materials.

engine, as may be the case in a "failed jet" or "choked jet" scenario. A failed jet means that a relativistic outflow that was launched by the central engine, but that its energy was insufficient for it to emerge well-collimated from the surface of the ejecta cloud.

\subsection{Dynamical features}

A wide engine scenario has been invoked in previous studies (Kasliwal et al. 2017; Gottlieb et al. 2018; Nakar et al. 2018). For the wide engine, the jet is slowed by its interaction with the merger ejecta, and eventually becomes quasi-spherical by the time it reaches the outskirts of the cloud (see Figure 5a).

An angular structure is formed in the wide engine scenario, as shown qualitatively in Figure 5 and quantitatively in Figure 6. Unlike the narrow engine scenario, the angular structure developing for the wide jet is found to deviate from the quasi-Gaussian model in Equation 1. Nevertheless, its profile may still be characterized an larger opening angle, which is larger $\theta_{c}=0.33$ than in the narrow jet case. Furthermore, the wide jet is found to have a lower peak isotropic equivalent energy
$E_{\text {iso,peak }}=4 \pi \epsilon_{0} \approx 9 \times 10^{51} \mathrm{erg}$ relative to the narrow engine model.

Whereas in the narrow engine model, roughly $20 \%$ of the jet energy is seen to be deposited in the merger cloud, we find that $80 \%$ of the jet energy is given to the merger cloud in the wide engine scenario. Figure 7 shows the dependence of the energy-averaged Lorentz factor (Equation 2) as a function of the polar angle, and at various epochs in the jet evolution. $\Gamma_{\text {avg }}$ ranges from 3 to 15 between polar angles of 0.0 and $0.4 \mathrm{rad}$.

\subsection{Afterglow light curve}

In Figure 9 we show broad-band afterglow light curves computed from the wide engine model, compared with observations at radio, optical, and X-ray frequencies. For this model, we were only able to obtain a successful fit with a very low external density of $n=10^{-5} \mathrm{~cm}^{-3}$.

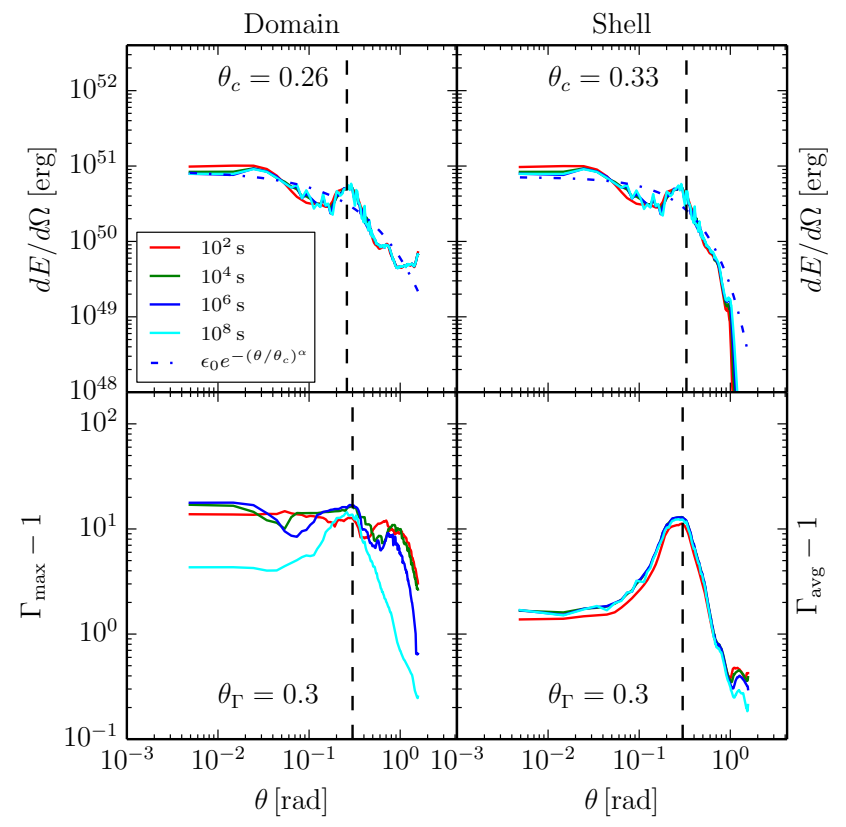

Figure 6. Wide engine model — Same as Figure 2, but for the wide engine model. Shown in the top row is the angular distribution of total energy (kinetic plus thermal) for the whole simulation domain (left panel) and the relativistic shell (right panel). The bottom row shows the maximum Lorentz factor over the whole simulation domain (left panel) and the energy averaged Lorentz factor for the relativistic shell (right panel). Results from different time snapshots are colored individually with $t_{\text {lab }}=10^{2} \mathrm{~s}$ (red), $10^{4} \mathrm{~s}$ (green), $10^{6} \mathrm{~s}$ (blue), $10^{8} \mathrm{~s}$ (cyan). The angular distribution profile of the total energy is well fitted by a quasi-Gaussian model $\epsilon_{0} e^{-\left(\theta / \theta_{c}\right)^{\alpha}}$. We use the fitting value from the relativistic shell: $4 \pi \epsilon_{0} \approx 9 \times 10^{51} \mathrm{erg}, \theta_{c}=0.33$, and $\alpha=1.07$. The wide engine model has a large half opening angle $\theta_{c}=0.33$, indicated by the vertical dashed purple line. 


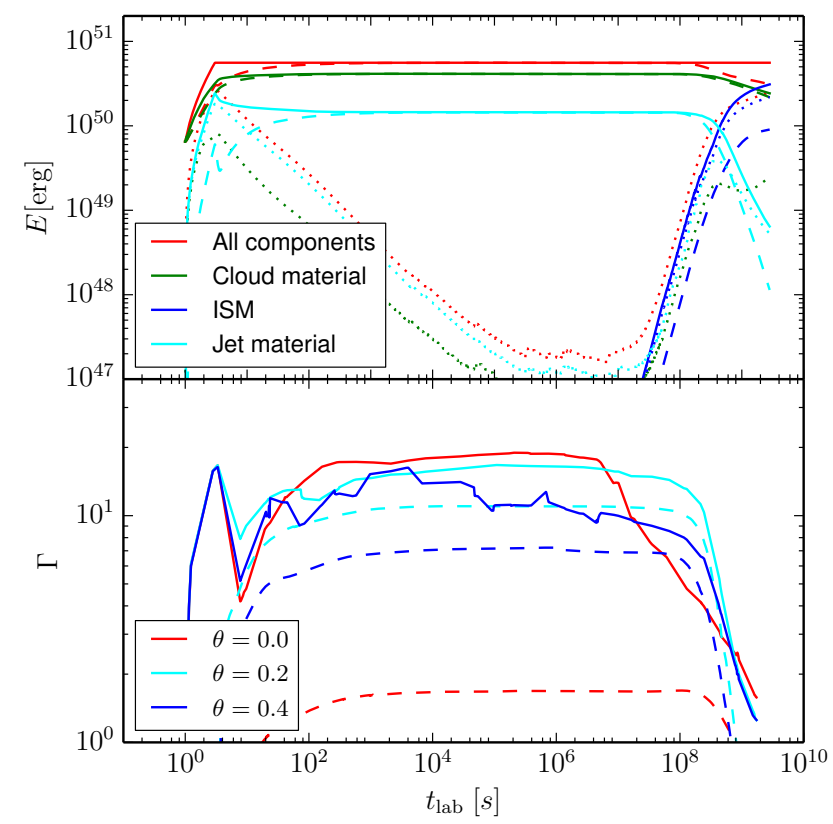

Figure 7. Wide engine model — Same as Figure 3 but for the wide engine simulation: a quasi-spherical jet propagating in the ISM environment with uniform density $n=$ $10^{-5} \mathrm{~cm}^{-3}$. In the top panel, solid lines represent the total energy (kinetic plus thermal) for four components: everything in the domain (red), the ejecta cloud (green), the ISM (blue), and the jet engine (cyan). Dashed lines (dotted lines) represent kinetic energy (thermal energy) for each component. In less than $10 \mathrm{~s}$, about $80 \%$ of the jet energy is transferred to the cloud. In the bottom panel, the time evolution of the maximum Lorentz factor along different polar angles is shown in solid lines. The energy-averaged Lorentz factor of the relativistic shell is shown in dashed lines. Compared to Figure 3 for the successful jet case, the Lorentz factor of the relativistic shell is moderate.

\subsection{Ejecta Lorentz factor distribution}

In the literature (e.g. Mooley et al. 2018), the stratified quasi-spherical explosion model utilizes an outflow profile: $E(>\Gamma \beta) \propto(\Gamma \beta)^{-\alpha}$. The energy power-law index value $\alpha=5$ has been found to match early observations ( $<100$ days). In the left column of Figure 8, we show the cumulative distribution function of energy $E(>\Gamma \beta)$ as a function of four velocity. For the wide engine model, we find that $E(>\Gamma \beta)$ is not well characterized by a single power-law. Rather, $\alpha$ increases from roughly 0.3 on the low velocity end and increases toward 1 at $\Gamma \beta \sim 10$ (qualitatively similar to results of Hotokezaka et al. 2018). This is in contrast with the narrow engine model we explored in Section 3, where a significant fraction of the energy was seen to reside at high Lorentz factor. The right column of Figure 8 shows the four velocity distribution histogram of the total energy and the thermal energy at $t_{\mathrm{lab}}=10^{8} \mathrm{~s}$.
A large amount of shock-generated thermal energy resides in the ultra-relativistic shell $(\Gamma>10)$ for the narrow engine model. The shock-generated thermal energy of the cloud and the jet engine material has higher Lorentz factor compared with the thermal energy of the shock-heated ISM.
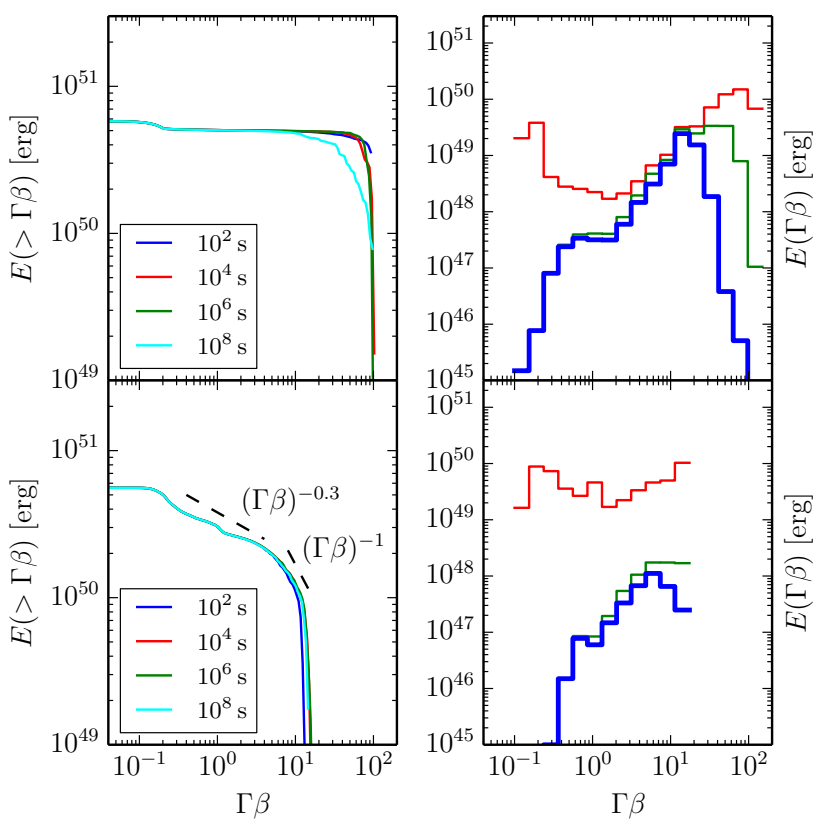

Figure 8. The energy four-velocity structure for the narrow engine (top row) and the wide engine model (bottom row). The energy four velocity cumulative plot (left column) shows that the outflow from the wide engine model is more stratified radially. In the wide engine model, slow moving materials contain a large fraction of the total energy. In the narrow engine model, most of the energy is contained in the ultra-relativistic shell instead. The right column displays the energy four velocity distribution histogram at $t_{\text {lab }}=10^{8} \mathrm{~s}$. During jet's transition from the coasting phase to the deceleration phase, the strong forward and reverse shock generates a large amount of thermal energy in the ISM, also in the entrained ejecta cloud (and the jet engine) materials. The red histogram represents the total energy (kinetic plus thermal) in the entire domain. The green histogram represents the total thermal energy. And the thick blue histogram shows the thermal energy in the ISM only.

\subsection{Light curve comparison between narrow and wide engines}

Off-axis light curves from the narrow engine model and both on-axis and off-axis light curves from the wide engine model are able to match the rising light curve observed in the first $\sim 100$ days of GRB170817A. The rising light curve component in all of these cases is produced by stratification. In the case of the narrow en- 


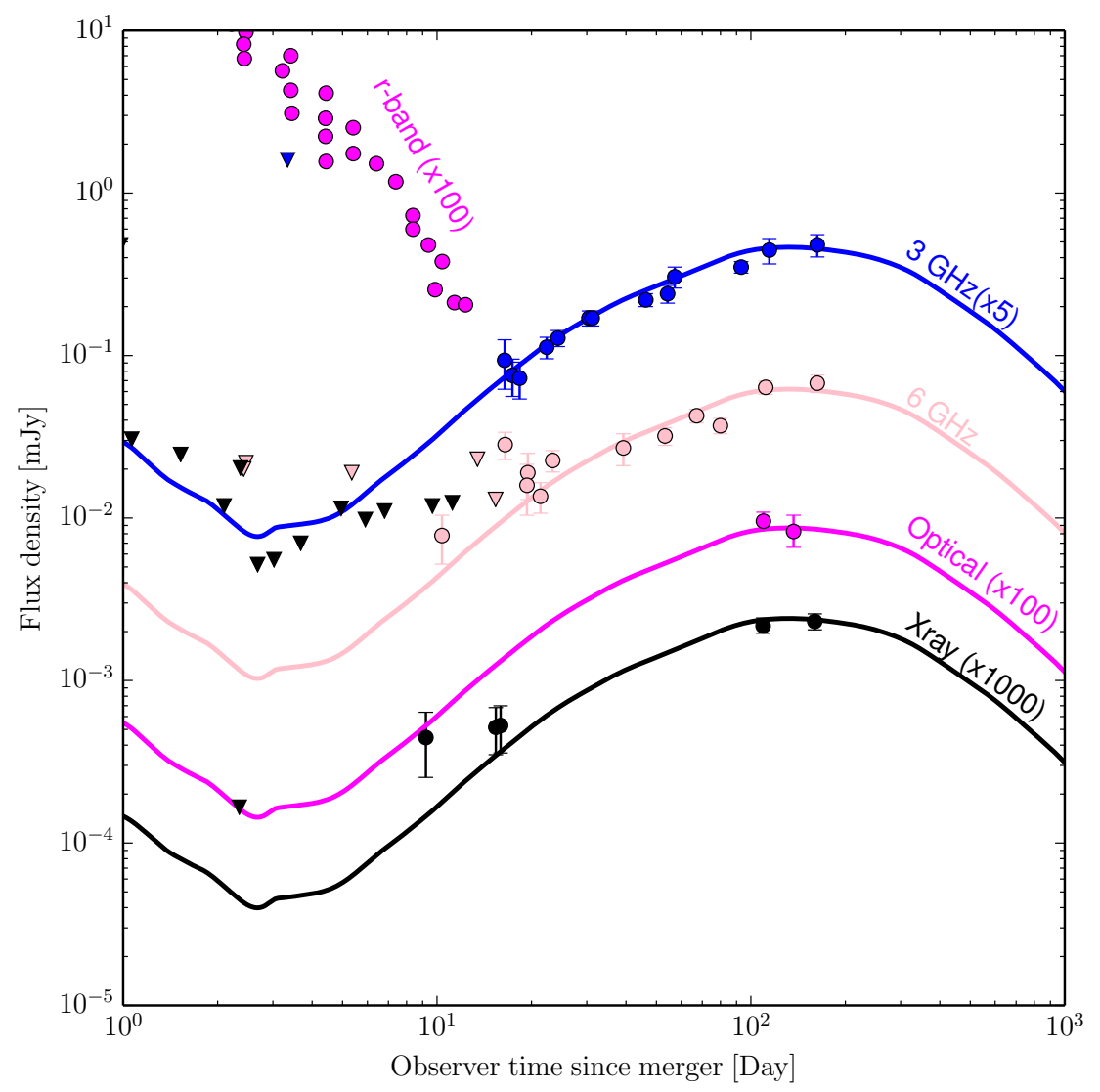

Figure 9. Wide engine model — The fitting on-axis light curve calculated from the numerical simulation of the mildly relativistic quasi-spherical outflow propagating in an ISM environment with uniform density $10^{-5} \mathrm{~cm}^{-3}$. The fitting radiation parameter values are $\theta_{\mathrm{obs}}=0, \epsilon_{e}=0.1, \epsilon_{B}=2 \times 10^{-3}$, and $p=2.16$. The presented multi-band light curves (smoothed by Savitzky-Golay filter) are consistent with both the early flux upper-limits for non-detection (down triangle) and detected signals (dots with error bar) in Radio $(3 \mathrm{GHz}, 6 \mathrm{GHz})$, Optical $\left(5 \times 10^{14} \mathrm{~Hz}\right)$ and X-ray $(1 \mathrm{keV})$. The non-detection upper-limits in Radio, X-ray and the non-thermal observations in Radio $(3 \mathrm{GHz}, 6 \mathrm{GHz})$, Optical $\left(5 \times 10^{14} \mathrm{~Hz}\right)$ and X-ray $(1 \mathrm{keV})$ are all taken from Margutti et al. (2018). The early "kilonova" r-band data taken from Lyman et al. (2018) are also presented for comparison. We refer the reader to Sections 5 and 6 for discussions about the early declining light curve.

gine model producing a successful stratified jet, angular stratification is of importance. The high latitude (i.e. near the axis) relativistic material decelerates first and adds smoothly to the rising light curve (see Section 5) without producing a sudden brightening when the jet core decelerates and comes into view for off-axis observers. Indeed, if an angularly structured jet is responsible for GRB170817A, the jet core is already being observed. In contrast, for the wide engine model producing a quasi-isotropic explosion the outflow is radially stratified with slower material catching up with the decelerating blast wave. In both cases, the light curve comes from the mildly relativistic material (Nakar \& Piran 2018; see discussion in Section 5). Both models predict that the afterglow light curve will decay $\sim 200$ days after the merger, and share roughly the same decay pattern.

\section{SUCCESSFUL STRUCTURED JET AND ITS MULTI-STAGE LIGHT CURVE}

Section 3 presented the dynamics and multi-band light curves from the successful structured jet simulation. Here we analyze the light curve features in detail, focussing on the X-ray light curve, and compare with analytic estimates. In order to post-process each simulation output in the time series of saved data files to compute synchrotron light curves, we first calculate the photosphere location by integrating the optical depth along the observer's line of sight given by:

$$
\tau=\int_{r_{p h}}^{\infty} \sigma_{T} \Gamma(1-\beta \cos \theta) n^{\prime} d l,
$$

where $\beta$ is the absolute value of the velocity normalized by the speed of light, $\theta$ is the angle between the velocity vector and line of sight, $d l$ is the distance along 


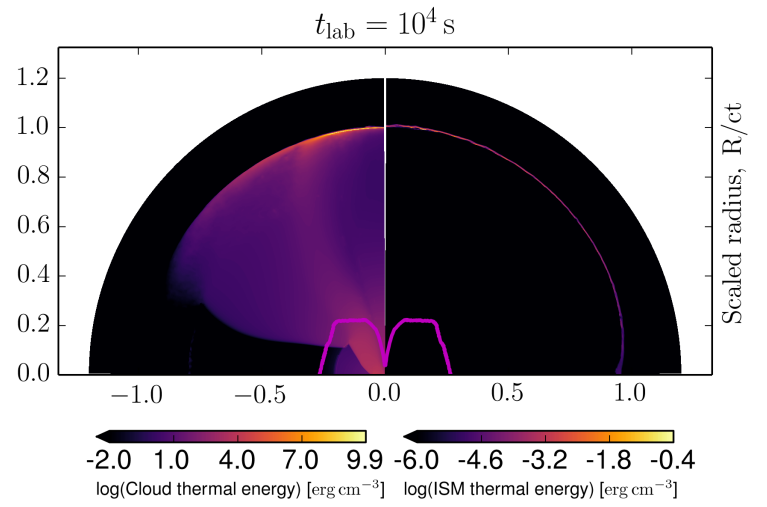

(a)

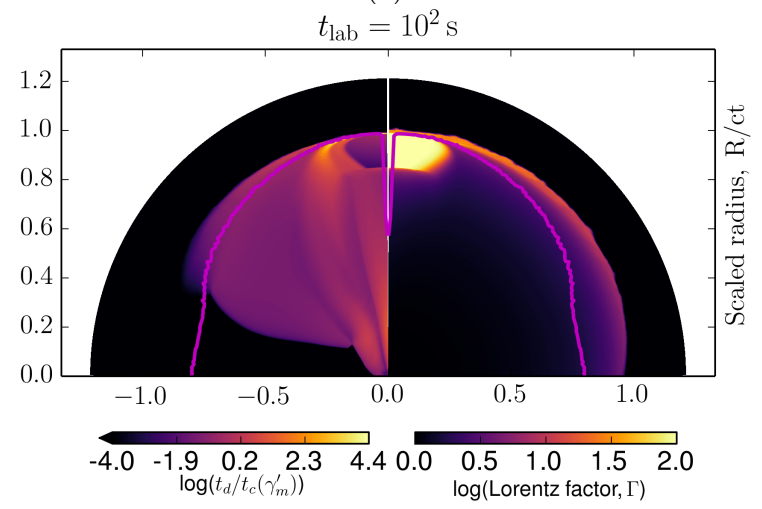

(b)

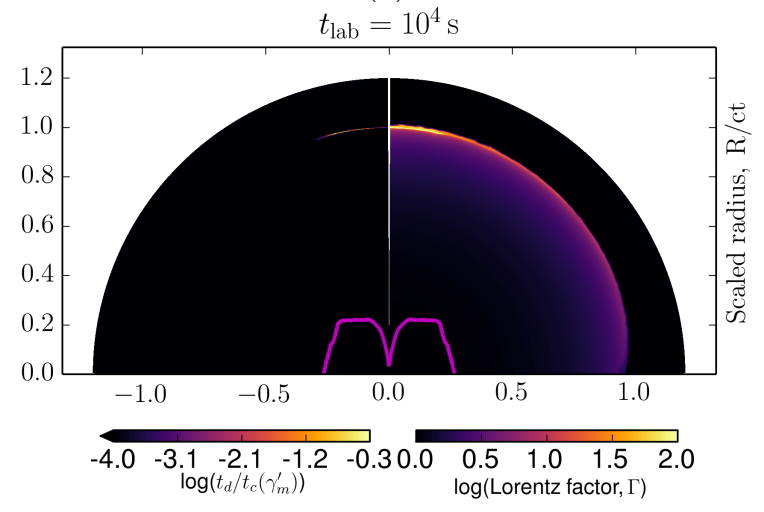

(c)

Figure 10. Narrow engine model - (a) The thermal energy contour plot of the merger ejecta (Left panel) and of the ISM (right panel). The contour snapshot is taken at $t_{\text {lab }}=$ $10^{4}$ s. (b) Left Panel: The ratio contour plot of the dynamical time $t_{d}$ versus the cooling time $t_{c}$ at $t_{\text {lab }}=10^{2} \mathrm{~s}$. Right Panel: the Lorentz factor contour plot at $t_{\text {lab }}=10^{2} \mathrm{~s}$. (c) Same with (b), but at a different time $t_{\text {lab }}=10^{4} \mathrm{~s}$. The magenta line indicates the photosphere position viewed by on-axis observers. During the time period $10^{2}-10^{4} \mathrm{~s}$, the emitting region (thin shell near the shock front) makes the transition from fast cooling to slow cooling.

line of sight, $n^{\prime}$ is the proper electron number density (Mizuta et al. 2011), and $\sigma_{T}$ is the Thomson cross section for electron scattering. The photosphere position corresponding to the $\tau \sim 1$ surface is used to identify optically thin regions of the simulation volume. The photosphere position for on-axis observers is shown in Figure 10. We calculate the synchrotron emissivity from the optically thin fluid in the simulation cells above the photosphere position to compute light curves.

To determine whether the electrons in a fluid element are in the fast cooling or slow cooling regime, we calculate the dynamical time $t_{d}$, the minimum Lorentz factor $\gamma_{m}^{\prime}$ of the electrons, and the associated cooling time $t_{c}\left(\gamma_{m}^{\prime}\right)$, according to:

$$
\begin{aligned}
t_{d} & =R /\left(c \Gamma^{2}\right) \\
\gamma_{m}^{\prime} & =\frac{(p-2) /(p-1) \epsilon_{e} e^{\prime}}{\left(\rho^{\prime} / m_{p}\right) m_{e} c^{2}}, \\
t_{c}\left(\gamma^{\prime}\right) & =3 m_{e} c /\left(4 \sigma_{T} \Gamma \gamma^{\prime} \epsilon_{B} e^{\prime}\right) .
\end{aligned}
$$

When the dynamical time exceeds the cooling time, $t_{d}>t_{c}\left(\gamma_{m}^{\prime}\right)$, the fluid element is in the fast cooling regime. At $t_{\text {lab }}>10^{2} \mathrm{~s}$, the fast cooling regions fall behind the photosphere and are thus not included in the synchrotron radiation calculation. By $t_{\text {lab }}=10^{4} \mathrm{~s}$, the entire simulation volume is in the slow cooling regime ${ }^{1}$ (see Figure 10b-10c).

\subsection{X-ray light curve; comparison with analytic estimates}

In Figure 11 we display the X-ray synchrotron emission light curve calculated from our narrow engine simulation. The light curve covers seven orders of magnitude in observer time starting from one minute and extending to $\sim 30$ years after the BNS merger. The microphysical parameters $\epsilon_{e}$, the relativistic electron fraction, $\epsilon_{B}$, the magnetic energy fraction, and $p$, the slope of the electron distribution are set to standard values: $\epsilon_{e}=0.1, \epsilon_{B}=0.01$, and $\mathrm{p}=2.2$. In order to check the accuracy of our numerical light curves, we compare the peak time and peak flux to estimates from existing analytical models, shown in Figure 11. The first model (Estimate_A) is based on an adiabatic double-sided top hat jet with total kinetic energy $E_{k}$, an initial opening angle $\theta_{j}$ and a simple hydrodynamical evolution model (Granot et al. 2017; Nakar et al. 2002). The peak time of the off-axis afterglow light curve occurs when the bulk Lorentz factor of the top hat jet drops to $\Gamma=1 / \theta_{\mathrm{obs}}$.

\footnotetext{
${ }^{1}$ In the radiation calculation we include the effect of electron cooling using a global estimate for the electron cooling time equal to the lab frame time since the BNS merger.
} 


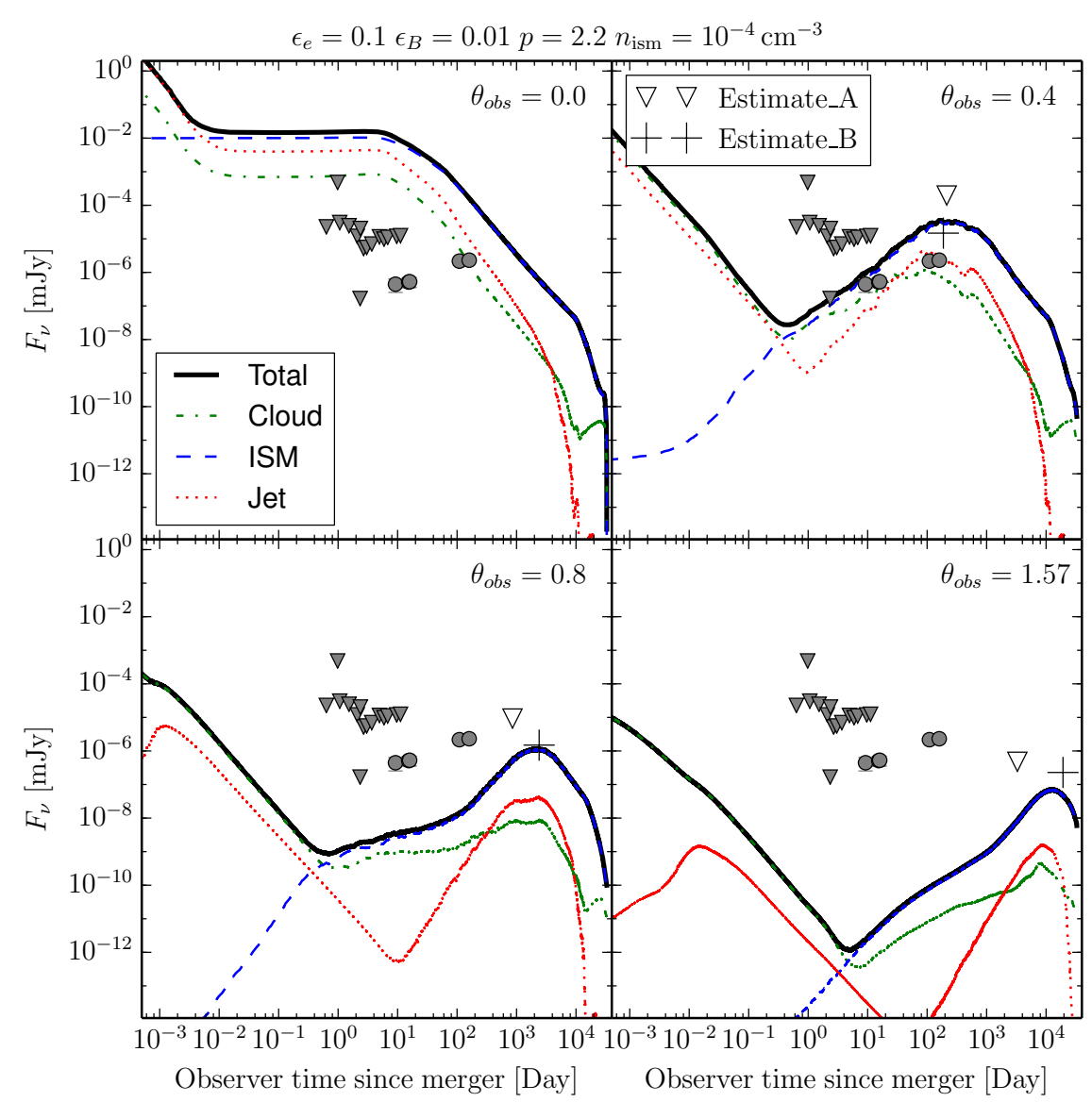

Figure 11. Narrow engine model - The X-ray $(1 \mathrm{keV})$ light curve at different observer angles with radiation parameter $\epsilon_{e}=0.1, \epsilon_{B}=0.01, p=2.2$ and ISM density $n=10^{-4} \mathrm{~cm}^{-3}$. Black solid lines represent the total synchrotron emission from the forward jet (radiation from the counter jet is not included). The flux contribution among the three components (the ejecta cloud, the ISM, the jet engine) are separated based on their mass fraction. Synchrotron radiation from the cloud/ism/jet is shown in green dot-dashed/blue dashed/red dotted line, respectively. The on-axis and off-axis light curve displays an universal feature: an initial rapidly declining followed by a late re-brightening. The early declining light curve has internal shock origin (i.e. from the cloud or the jet engine material). The late re-brightening light curve comes from the external shock (i.e. from the shocked ISM). The peak time and the peak flux of the late re-brightening light curve well matches the analytical estimation. Two estimation methods are being used here: Estimate_A model (hollow down triangle) from (Nakar et al. 2002; Granot et al. 2017), and Estimate_B model (plus) from (Lamb \& Kobayashi 2017a). We refer the reader to Section 6 for the viability discussion of the early declining light curve.

The peak time and the peak flux are given as

$$
\begin{aligned}
t_{\text {peak }}\left(\theta_{\text {obs }}\right) & =0.7(1+z)\left(\frac{E_{\mathrm{k}, 51}}{n_{0}}\right)^{1 / 3}\left(\frac{\theta_{\text {obs }}}{0.1}\right)^{2} \text { days },(10) \\
F_{\nu_{m}<\nu<\nu_{c}}^{\text {peak }}(t) & =0.6 \frac{g_{1}(p)}{g_{1}(2.2)}(1+z)^{(3-p) / 2} D_{L 28}^{-2} \\
& \times \epsilon_{e,-1}^{p-1} \epsilon_{B,-2}^{\frac{p+1}{4}} n_{0}^{\frac{p+1}{4}} E_{\mathrm{k}, 50.7} \nu_{14.7}^{(1-p) / 2} \theta_{\text {obs },-1}^{-2 p} \text { mJy } .
\end{aligned}
$$

In another model (Estimate_B), the projected surface area and the solid-angle of emission are taken into consideration (Lamb \& Kobayashi 2017a). The peak time and the peak flux are given by

$$
\begin{aligned}
t_{\text {peak }}\left(\theta_{\text {obs }}\right) & =195\left[\frac{(5+p)(7-p)^{1 / 3}}{(p-1)^{4 / 3}}\right]\left(\theta_{\text {obs }}-\theta_{j}\right)^{8 / 3} \\
& \times n_{-1}^{-1 / 3} E_{\mathrm{k}, 52}^{1 / 3} \text { days } \\
f_{\nu_{m}<\nu<\nu_{c}}^{\text {peak }} & =C(p) f\left(\theta_{\text {obs }}, \theta_{j}\right)\left(\theta_{\text {obs }}-\theta_{j}\right)^{2(1-p)} \nu^{(1-p) / 2} \\
& \times E_{k} n^{(1+p) / 4} \epsilon_{B}^{(1+p) / 4} \epsilon_{e}^{p-1} D_{L}^{-2} \operatorname{erg~s}^{-1} \mathrm{~cm}^{-2} \mathrm{~Hz}^{-1}
\end{aligned}
$$

where the expressions for $g_{1}(p), C(p), f\left(\theta_{\text {obs }}, \theta_{j}\right)$ are given in Granot et al. (2017) and Lamb \& Kobayashi (2017a) and $E_{k}=E_{\text {iso }} \theta_{j}^{2} / 2$ is the jet kinetic energy (double sided). Here we model the ultra-relativistic core of the structured jet simply as a uniform top-hat jet with kinetic isotropic equivalent energy $E_{\text {iso, } 52} \sim 6$, and jet 


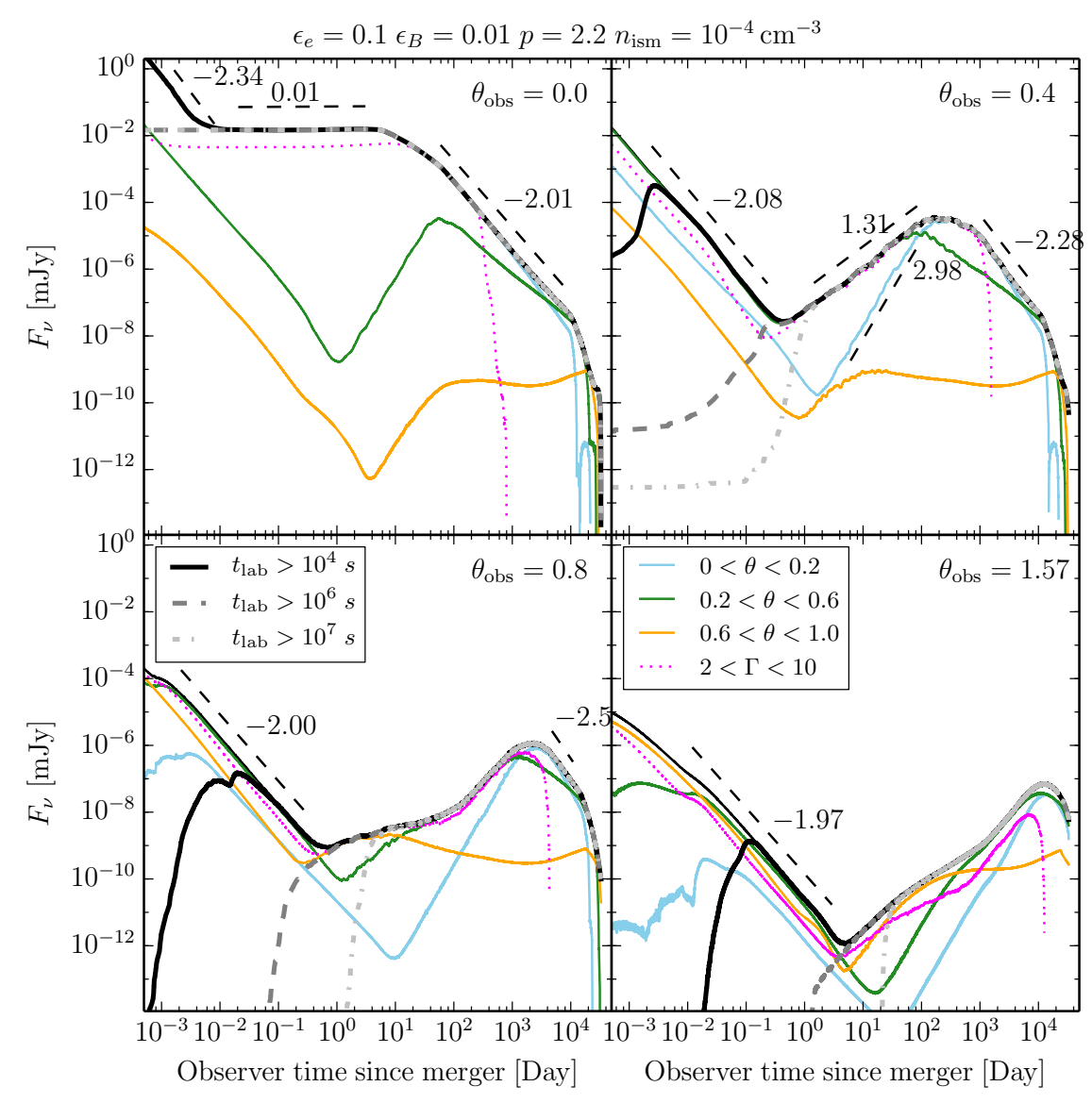

Figure 12. Narrow engine model - The temporal and spatial flux contribution for the on-axis and off-axis X-ray (1 keV) light curve. The post-prompt light curve exhibits three stages: an early afterglow, an intermediate transition, and a late afterglow. The black thick solid lines display the total flux emitted by fluid elements during the time period $t_{\text {lab }}>10^{4} \mathrm{~s}$, measured in central lab frame. Gray thick dashed lines (silver thick dot-dashed lines) represent a different time period: $t_{\text {lab }}>10^{6} \mathrm{~s}\left(t_{\text {lab }}>10^{7} \mathrm{~s}\right)$. The early afterglow part is emitted before $t_{\text {lab }}=10^{6} \mathrm{~s}$. Both the intermediate transition and the late afterglow come from a time period $t_{\text {lab }}>10^{6} \mathrm{~s}$ (see also Figure 3). The synchrotron emission from different angular regions in the domain is shown in thin solid lines. The blue/green/orange line shows the flux contributed by fluid elements within a domain lateral angle extending from 0.0/0.2/0.6 to 0.2/0.6/1.0 [rad] (flux from the fluid with domain angle larger than $1.0 \mathrm{rad}$ is minimal and not presented here). The early afterglow and the intermediate transition light curve for off-axis observers initially comes from the angular region closer to the line of sight. The late afterglow comes from the central angular region $0<\theta<0.2$. The magenta dotted line displays the flux contributed by fluid elements with Lorentz factor larger than 2, but smaller than 10 . All of the observed emission before the observer time $t_{\mathrm{obs}} \sim 200$ days originates from the relativistic thin shell with Lorentz factor larger than 2 (except for $\theta_{\mathrm{obs}}=1.57$ ). Part of the late afterglow comes from the decelerated sub-relativistic materials.

half opening angle $\theta_{j} \sim 0.15$. The ISM density is set to the value adopted in the simulation, $n=10^{-4} \mathrm{~cm}^{-3}$. As shown in Figure 11, the analytical estimate of the peak time and the peak flux at different viewing angles from Estimate_B is in agreement with the calculated light curve.

\subsection{X-ray light curve shape}

The on-axis X-ray light curve shown in Figure 12 (top left panel) displays three temporal power-law segments: 1) an early time steep decay phase $F_{\nu} \propto t^{-\alpha}$, with temporal index $\alpha_{1} \sim 2.3$. 2) a shallow decay (plateau) phase with index $\alpha_{2} \sim 0$, and 3) a later decay phase with index $\alpha_{3} \sim 2$. These light curve components share similarities with the on-axis X-ray light curves for GRBs observed by Swift (Zhang et al. 2006; Kumar \& Zhang 2015) but with a compressed time scale.

The off-axis light curves shown in Figure 12 (top right and bottom two panels) exhibit an early rapidlyfading phase followed by a later re-brightening. Both on-axis and off-axis light curves have three common stages: an early declining afterglow, an intermediate transition phase (the rising part), and a late afterglow (the late declining part). The early declining emission mainly comes from the shock-heated cloud (for the onaxis light curve, it is the jet instead) and decays on a 
time scale of minutes to days depending on the viewing angle. The flux contribution from the external shock in the ISM steadily increases. Both the intermediate transition phase and the late afterglow light curve come from the shock heated ISM (see Figure 11).

\subsection{Temporal decomposition of the light curve}

Figure 12 shows the temporal and spatial decomposition of the computed light curve. First, we separate the entire simulation duration into three lab time periods: $t_{\text {lab }}>10^{4} \mathrm{~s} ; t_{\text {lab }}>10^{6} \mathrm{~s} ; t_{\text {lab }}>10^{7} \mathrm{~s}$. Most of the early declining flux observed during the first $\sim$ day of observer time is emitted during the lab frame time period $10^{4} \mathrm{~s}<t_{\text {lab }}<10^{6} \mathrm{~s}$ for both on-axis and offaxis light curves. As seen in Figure 3, before lab time $t_{\text {lab }} \sim 10^{7} \mathrm{~s}$, almost all of the thermal energy in the domain is in the jet engine and the merger cloud material. The early declining emission is due to the cooling of the post-shock jet engine and merger cloud material. At later times, this material accumulates into a relativistic shell that experiences strong forward and reverse shocks as it sweeps up and shocks the ISM. The internal energy from the shock-heated ISM then begins to play an important role in the synchrotron emission. The flux during the intermediate transition and the late afterglow is mainly emitted during the lab time period $t_{\text {lab }}>10^{7} \mathrm{~s}$, consistent with the dynamical evolution of the thermal energy. The turning point between the early declining and the intermediate transition phases depends on the Lorentz factor of the emitting shell. For on-axis observers, photons radiated at lab frame time $t_{\text {lab }}$ and lab frame position $\boldsymbol{r}$ will reach an observer at observer time $t_{\text {obs }}$ (see e.g. Piran 1999; Mészáros 2006)

$$
t_{\mathrm{obs}}=(1+z)\left(t_{\mathrm{lab}}-\boldsymbol{r} \cdot \boldsymbol{n} / c\right) \sim(1+z) t_{\mathrm{lab}} / 2 \Gamma^{2} .
$$

where $\boldsymbol{n}$ is a unit vector pointing in the direction toward the observer. Along the jet propagation direction, the bulk Lorentz factor of the on-axis relativistic shell is $\sim$ 100. A photon emitted from the shell at lab time $t_{\text {lab }} \sim 10^{7} \mathrm{~s}$ will thus be received by on-axis observers at observer time $t_{\mathrm{obs}} \sim 8 \mathrm{~min}$. This determines the turning point between the initial steep decay and the shallow decay phase of the on-axis light curve shown in Figures 11 and 12 .

Previous studies suggest that the initial steep decay phase of the on-axis light curve is linked to the tail of the GRB prompt emission, and has internal shock origin (e.g. Barthelmy et al. 2005; Duffell \& MacFadyen 2015). Our result supports this interpretation. We refer the reader to Section 6 for further discussion about the early declining light curve. The shallow decay phase has been previously interpreted in the context of a refreshed shock model (Rees \& Mészáros 1998; Sari \& Mészáros 2000; Zhang et al. 2006). Based on our simulation results, we find the duration of the shallow decay phase depends on the initial bulk Lorentz factor and the isotropic equivalent energy of the relativistic jet. It also depends on the ambient density. The typical time scale of the plateau phase observed for classical GRBs is $10^{3} \sim 10^{4} \mathrm{~s}$ (Kumar \& Zhang 2015). For the BNS case considered here, an energetic jet propagating in a very low density environment results in a duration longer than this.

\subsection{Angular decomposition of the light curve}

For the off-axis light curve, the early rapidly-fading and later re-brightening behavior distinguishes it from the on-axis light curve. In Figure 12 we divide the simulation domain into angular regions and calculate the flux contribution from each of them. Off-axis observers will first detect radiation from the part of the outflow that is moving toward the observer, i.e. in the direction of the observer's line of sight. As time goes on, the decelerated relativistic shell at higher latitudes contributes to the re-brightening light curve at lower latitudes, driving the flux level smoothly to greater values (e.g. Lazzati et al. 2017b).

The early re-brightening portion of the light curve comes from off-axis mildly relativistic material moving along the line of sight toward the observer and is essentially "on-axis" emission from those fluid elements with respect to the observer. At $\theta_{\mathrm{obs}}=0.4$, the slope of the re-brightening light curve from the $0.2<\theta<0.6$ region is moderate $\sim 1.3$ (top right panel) while the slope of the re-brightening light curve from the $0<\theta<0.2$ region is significantly larger $\sim 3$. The difference in the slope value results from whether the light curve is observed "on-axis" or "off-axis" with respect to the line of sight. Observers located outside of the beaming cone of the relativistic shell $\theta_{\mathrm{obs}}>1 / \Gamma$, will see an "offaxis" light curve. The observed "off-axis" light curve should rise faster than $t_{\text {obs }}^{3}$ (Nakar \& Piran 2018). For the GW170817 BNS merger event, the fact that the observed multi-band light curve is much shallower, scaling as $F_{\nu} \propto t_{\mathrm{obs}}^{0.78}$, implies that "on-axis" emission was always observed for this event (Nakar \& Piran 2018).

For the structured jet model, that "on-axis" emission comes from the mildly relativistic sheath at an off-axis angle $\theta_{\text {obs }} \sim 20^{\circ}$. The energy-averaged Lorentz factor at this angle is around $\Gamma \sim 3$ (Figure 2, lower right panel), in agreement with the analytical constraint, $\Gamma \sim 1.5-7$, from Nakar \& Piran (2018). When the central ultrarelativistic core decelerates and become "on-axis", the light curve stops increasing and smoothly turns over. The peak flux is determined by the central relativistic 
core. This is consistent with the peak time and the peak flux estimates discussed in Section 5.1.

A similar re-brightening feature occurs in the observations of short GRBs (see e.g. Campana et al. 2006; Gao et al. 2015), long GRBs (e.g. Margutti et al. 2010), and X-ray Flashes (e.g. Huang et al. 2004). The analysis of the off-axis light curve made here may provide an alternative interpretation for those re-brightening events.

\section{POSSIBILITY OF A NON-THERMAL X-RAY "MERGER FLASH"}

It has been recognized (Nakar \& Piran 2017; Piro \& Kollmeier 2018) that shock-heating of the merger cloud by the relativistic jet may produce an observable thermal optical or UV flash at early times (minutes to hours) following the merger. Our hydrodynamic simulations are in overall agreement with this picture. We observe significant heating of the merger ejecta, resulting from a strong shock wave that is launched when the relativistic jet emerges from the cloud. This shock heating episode occurs at high optical depth, roughly $2 \times 10^{11} \mathrm{~cm}$ from the merger center (see Section 3). A majority of the shock-heated merger ejecta expands laterally, and is expelled significantly off-axis from the relativistic core of the jet, forming the angular structure also discussed in Section 3. The shock-heated material reaches temperatures on order $10^{7} \mathrm{~K}$, and accelerates to a Lorentz factor of $\sim 2$. This material becomes optically thin after expanding to a radius $\sim 10^{12}-10^{13} \mathrm{~cm}$, at which point the temperature has decreased adiabatically to $10^{3}-10^{4} \mathrm{~K}$. If radiating thermally, this material would produce a detectable UV flash, delayed from the GW chirp by several minutes, cooling and fading from view over the course of $\sim$ hours.

Here we discuss the possibility that the shock-heated material might instead radiate non-thermally. This would shift the emission to higher energies, potentially rendering it detectable by Swift XRT or even Fermi GBM, as well as future proposed wide-field X-ray detectors. Non-thermal emission from shock-heated merger ejecta could be easily differentiated from the early afterglow signal, because it is declining due to adiabatic expansion, whereas emission from the external shock is brightening.

Here we briefly discuss the likelihood of detecting an early X-ray flash from BNS mergers, assuming the emission from the shocked cloud material is non-thermal and thus appropriately treated by our synchrotron radiation model described in Section 5 .

\subsection{Detectability of an X-ray merger flash}

The early light curves (at observer times between minutes and hours following the GW chirp) shown in Figure
13 are computed with the synchrotron model, applied to the optically thin shock-heated merger ejecta. The early emission (hereafter a merger flash) decreases in time because of adiabatic cooling of the previously accelerated electrons. The flash is overtaken in all wave bands by rising synchrotron radiation from the external shock after roughly a day.

For the GW170817 BNS merger event, any early declining phase has been missed. The optical flux of the early synchrotron radiation is faint compared to the observed kilonova optical data (e.g., R-band). Early X-ray emission at several hours is below the instrument detection limit of Chandra. Figure 13 displays the detection limits of various instruments along with the observational data, and two sets of fitting light curves.

In X-ray, the late-XRT observations use a detection limit of $2 \times 10^{-14} \mathrm{erg} \mathrm{cm}^{-2} \mathrm{~s}^{-1}$ (for a $10 \mathrm{ks}$ exposure). For early-XRT, the detection limit is assumed to scale with the square root of the exposure time. For Chandra, we adopt a constant detection limit of $10^{-15} \mathrm{erg} \mathrm{cm}^{-2} \mathrm{~s}^{-1}$. In Figure 13, the X-ray detection limits have been converted to the flux limits in units of mJy assuming the default X-ray photon energy is $1 \mathrm{keV}$. In the optical, R-band imaging detection limit for HST is set to 27. In the radio, the detection limit of VLA is set to $10 \mu \mathrm{Jy}$, assuming a $10 \mathrm{~h}$ reaction time.

The associated early X-ray light curve would be detectable by Swift XRT until about 30 minutes following the GRB prompt emission. The hard X-ray light curve $(15 \mathrm{keV})$ becomes barely detectable by Swift BAT and Fermi GBM after one minute ${ }^{2}$. However, under favorable conditions, the detection of the early declining afterglow in radio, optical, X-ray at large off-axis angles may be possible for nearby BNS mergers.

\subsection{Distinguishing between successful jet and quasi-isotropic explosion from early $X$-ray emission}

As seen in Figures 4 and 9 both the narrow and wide engine models are capable of producing the late $\left(t_{\mathrm{obs}} \gtrsim 1\right.$ day) afterglow emission of GRB170817A. However, as shown in Figure 14, the non-detection of X-ray emission following GRB170817A by Fermi GBM on minutes to hours timescales may disfavor the wide engine model, because even if seen at $20^{\circ}$ off axis, this type of quasiisotropic explosion would have been detected by GBM at $\sim 15 \mathrm{keV}$ for $\sim$ minutes. Non-detection of the $\mathrm{X}$ -

\footnotetext{
${ }^{2}$ We take the $15-150 \mathrm{keV}$ band sensitivity of Swift BAT and Fermi GBM and divide it by the corresponding frequency of photon energy $15 \mathrm{keV}$ and $150 \mathrm{keV}$. This gives an approximation to the flux detection limits of these two instruments.
} 


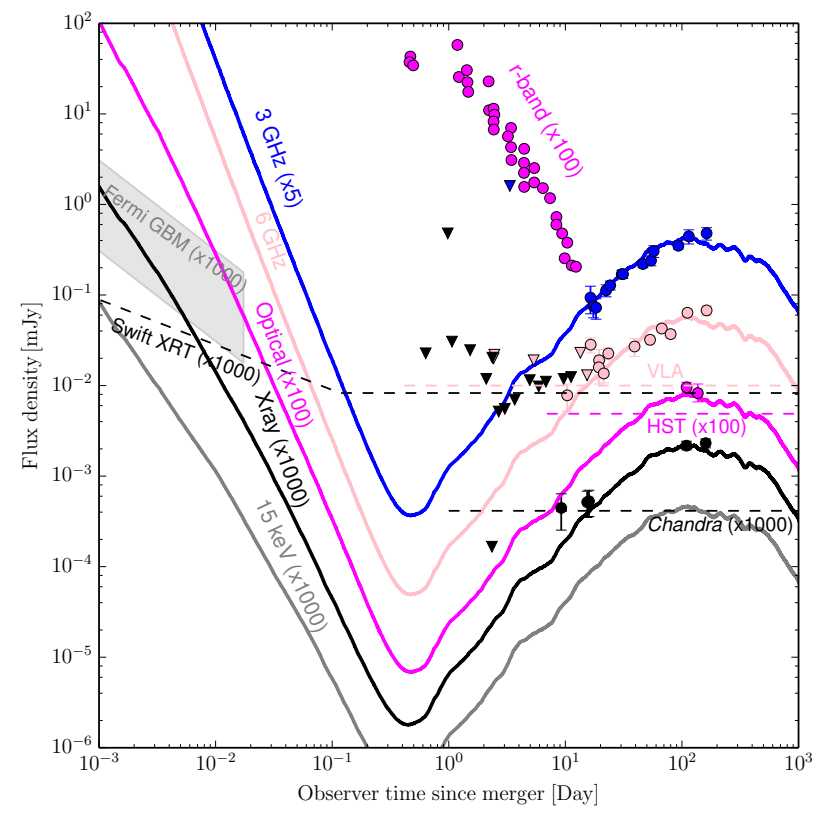

(a)

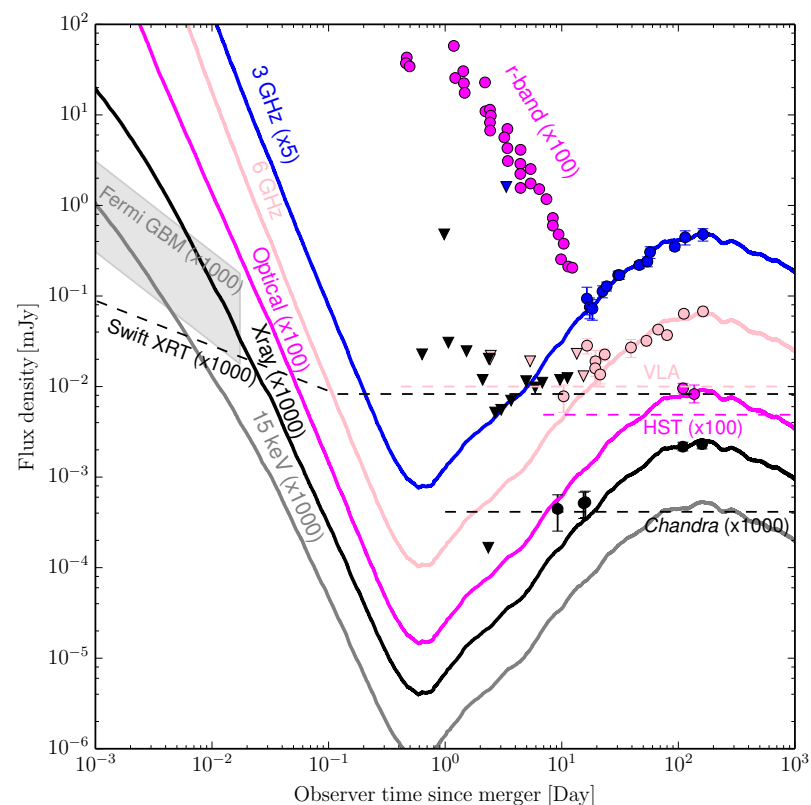

(b)

Figure 13. Narrow engine model - Similar to Figure 4. The plot shows two sets of fitting light curves calculated from the simulation of a structured jet propagating in an uniform ISM environment. In the left panel (a), the ISM density is $10^{-4} \mathrm{~cm}^{-3}$. The fitting radiation parameter values are $\theta_{\mathrm{obs}}=0.34\left(19.5^{\circ}\right), \epsilon_{e}=0.02, \epsilon_{B}=10^{-3}$, and $p=2.16$. In the right panel (b), the ISM density is $10^{-5} \mathrm{~cm}^{-3}$. The fitting radiation parameter values are $\theta_{\mathrm{obs}}=0.3\left(17^{\circ}\right), \epsilon_{e}=0.1, \epsilon_{B}=5 \times 10^{-4}$, and $p=2.16$. The light curves shown here are not smoothed by Savitzky-Golay and are extended to the early observer time starting from $\sim 1$ min. One added light curve (grey solid line) represents the flux of hard X-ray (15 keV), which is not (left panel) or barely (right panel) detectable by Swift BAT and Fermi GBM. The thresholds of their sensitivity are shown in the shaded region. We present the fitted multi-frequency light curves along with the detection limits of Swift-XRT (black), Chandra (black), HST (magenta), and VLA (pink). Swift-XRT is a promising tool to detect the early declining light curve if the BNS merger site has been found within an hour or so. We calculate the flux assuming the luminosity distance is $40 \mathrm{Mpc}$.

ray merger flash by GBM also favors the higher density $\left(n \sim 10^{-4} \mathrm{~cm}^{-3}\right)$ over a lower density $\left(n \sim 10^{-5} \mathrm{~cm}^{-3}\right)$ ISM. This is because in the wide jet scenario, resulting in a quasi-isotropic explosion, fitting the observed late afterglow light curve with a lower density ambient medium requires larger values of both $\epsilon_{B}$ and $\epsilon_{e}$. Such high values would place the X-ray merger flash within the detection threshold of GBM, in disagreement with the $\lesssim 2$ s duration of the GBM signal.

GW170817 occurred in a part of the sky not accessible to Swift due to Earth occultation. However, had this event been accessible to the Swift satellite and XRT had slewed to its location within minutes we show in Fig 14 that it could have detected a declining merger flash at $\sim 1 \mathrm{keV}$ lasting for $\sim$ minutes.

Future BNS merger detections are expected to occur more frequently at larger distances, $\gtrsim 100 \mathrm{Mpc}$. Had GRB170817A occurred at that distance, rather than $40 \mathrm{Mpc}$, it would not have triggered the GBM. Therefore, it is important to understand what other types of electromagnetic transients might be detectable from BNS mergers at larger distances.

\subsection{Applicability of the synchrotron emission model}

The emission model used to create the synthetic light curves in 13 and 14 assumes the presence of synchrotron radiating non-thermal electrons. For it to be applicable, we require a mechanism to produce and sustain the nonthermal electron population, $\epsilon_{e} \lesssim 0.1$. We must also invoke the presence of magnetic energy not too far below thermal equipartition, $\epsilon_{B} \lesssim 10^{-2}$.

Non-thermal electrons may be Fermi-accelerated at the sub-photospheric shock that forms around the time the jet breaks out from the merger cloud, at a distance of $\sim 2 \times 10^{11} \mathrm{~cm}$. They might also be accelerated by magnetic reconnection operating in the outflowing material, if a sufficient level of magnetic field energy was present in merger environment.

Sub-equipartition level magnetic fields may be produced downstream of the internal shock via the Weibel instability (although this depends on uncertain kinetic physics of radiation mediated shocks). Magnetic energy 


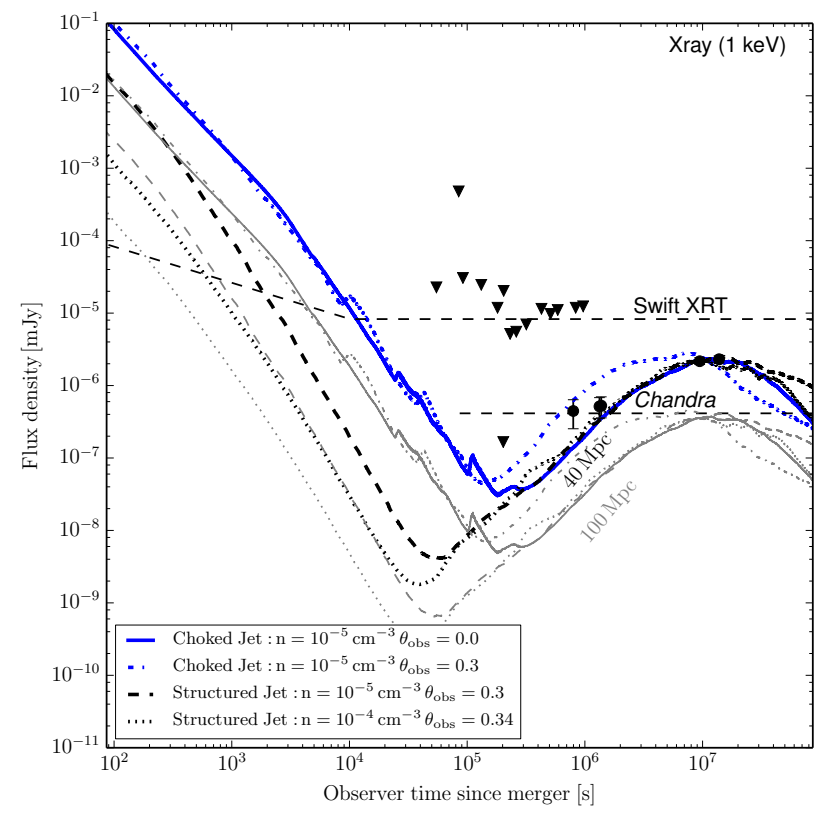

(a)

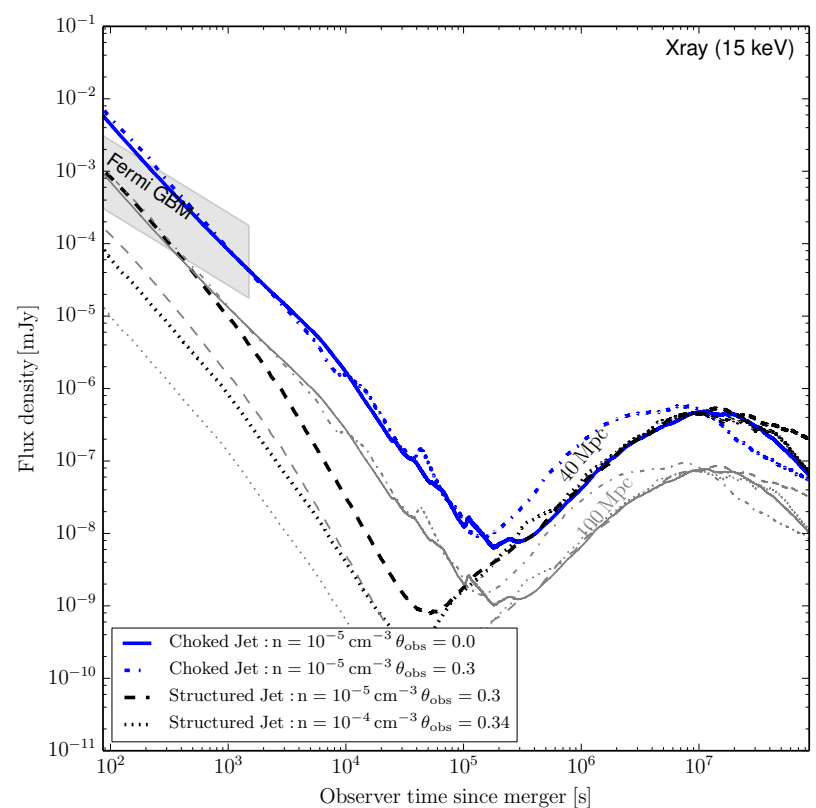

(b)

Figure 14. The comparison of the soft X-ray (1keV) light curve (left panel) and hard X-ray (15 keV) light curve (right panel) calculated from the two structured jet simulations (thick black lines) and one wide engine simulation (thick blue lines) performed in this study. The fitting radiation parameter values are listed in the captions of Figures 4 and 9. For the wide engine model, we also include the X-ray light curve at an off-axis viewing angle $\theta_{\text {obs }}=0.3$ (blue dot-dashed line) with the same microphysical parameter values adopted in the calculation of on-axis light curves. The light curves shown here are not smoothed by SavitzkyGolay and are extended to the early observer time starting from $\sim 1 \mathrm{~min}$. Two sets of comparison light curves are shown in the plot, corresponding to the same source observed at $40 \mathrm{Mpc}$ (thick lines) and $100 \mathrm{Mpc}$ (thin grey lines), respectively. The flux magnitude of the early declining light curve from the on-axis wide engine model is significantly higher than off-axis structured jet models. The turning point in the light curve depends on the viewing angle and the ISM density.

might also exist in the neutron star merger ejecta from either the pre-merger neutron star magnetic field, or dynamo amplification during the merger itself (Zrake \& MacFadyen 2013). Although magnetic energy density decreases as the merger ejecta expands, $\epsilon_{B}$ does not evolve significantly. This is because the energy density of the tangential magnetic field $\left(B_{\phi}\right.$ and $\left.B_{\theta}\right)$ in the coasting shocked cloud decreases like $r^{-2}$, while the gas internal energy decreases like $r^{-2 \gamma}$ where the adiabatic index is $\gamma \geq 4 / 3$. Therefore under expansion alone, $\epsilon_{B}$ either stays the same or marginally increases with radius.

\section{CONCLUSIONS AND DISCUSSION}

In this study we have presented moving-mesh, relativistic hydrodynamic simulations to explore the dynamics and radiative signatures of merging neutron star outflows. We have focused our modeling on two primary scenarios, dubbed the narrow and wide engine models. These scenarios represent different distributions of the relativistic material injected by a central engine that forms as a result of the neutron star coalescence. The narrow engine model is well collimated, and penetrates the debris cloud surrounding the merger site, propagating successfully into the circum-merger medium. This successful jet may be observed as a classical short gamma-ray burst by observers located along its axis. In contrast, the wide engine model fails to break out of the merger cloud, and instead drives a quasispherical shock through the cloud and into the surrounding medium.

Both the narrow and wide engine models can explain the afterglow of GRB170817A, including observations through $\sim 200$ days after the GW signal (Figures $4 \&$ 9 ). We find that in both scenarios, the jet develops an angular structure as a result of its interaction with the merger ejecta cloud. Both models predict the afterglow light curve to begin decaying after $\sim 200$ days, in a similar manner. Thus, upcoming observations of the late afterglow emission may not resolve the question of which scenario was the case for the GW170817 BNS merger event. Similar conclusions are made in (Margutti et al. 2018; Nakar \& Piran 2018).

However, as we discussed in Section 6.2, we surmise that non-detection of longer-lived $(\sim$ minutes) X-ray 
emission by GBM disfavors the wide engine model. Instead, the narrow engine model is favored because it can be fit to the late afterglow light curve without overpredicting the magnitude of an early X-ray flash. As discussed in Section 6.3, these conclusions are dependent on the presence of synchrotron radiating non-thermal particles in the mildly relativistic shocked neutron star material. Hence, the detection of an X-ray merger flash is potentially valuable as a probe of previously unexplored plasma conditions. In particular, its existence would indicate that either electrons are accelerated by sub-photospheric, radiation-mediated shocks, or by sustained dissipation of magnetic energy as the shell expands.

Previous studies have also considered the radiative signatures of structured jets (Lazzati et al. 2017a,b; Kathirgamaraju et al. 2018; Lyman et al. 2018; Troja et al. 2018; Lamb \& Kobayashi 2017b). In this work we have conducted simulations starting from the scale of the engine and continuing self-consistently to the afterglow stage. These engine-to-afterglow simulations reveal that jet structures that are consistent with the observations are a natural consequence of the hydrodynamical interaction of the jet with the environment of merging binary neutron stars.

This research was supported in part by the National Science Foundation under Grant No. AST-1715356. 


\section{REFERENCES}

Abbott, B. P., Abbott, R., Abbott, T. D., et al. 2017, ApJL, 848, L12

Alexander, K. D., Berger, E., Fong, W., et al. 2017, ApJL, 848, L21

Barthelmy, S. D., Cannizzo, J. K., Gehrels, N., et al. 2005, ApJL, 635, L133

Berger, E. 2014, ARA\&A, 52, 43

Blandford, R. D., \& McKee, C. F. 1976, Physics of Fluids, 19,1130

Campana, S., Tagliaferri, G., Lazzati, D., et al. 2006, A\&A, 454,113

Chornock, R., Berger, E., Kasen, D., et al. 2017, ApJL, 848, L19

Coulter, D. A., Foley, R. J., Kilpatrick, C. D., et al. 2017, Science, 358, 1556

Cowperthwaite, P. S., Berger, E., Villar, V. A., et al. 2017, ApJL, 848, L17

D'Avanzo, P., Campana, S., Ghisellini, G., et al. 2018, ArXiv e-prints, arXiv:1801.06164

De Colle, F., Granot, J., López-Cámara, D., \&

Ramirez-Ruiz, E. 2012, ApJ, 746, 122

Dobie, D., Kaplan, D. L., Murphy, T., et al. 2018, ArXiv e-prints, arXiv:1803.06853

Duffell, P. C., \& MacFadyen, A. I. 2013, ApJ, 775, 87

-. 2015, ApJ, 806, 205

Duffell, P. C., Quataert, E., \& MacFadyen, A. I. 2015, ApJ, 813,64

Fong, W., Berger, E., Margutti, R., \& Zauderer, B. A. 2015, ApJ, 815, 102

Gao, H., Ding, X., Wu, X.-F., Dai, Z.-G., \& Zhang, B. 2015, ApJ, 807, 163

Goldstein, A., Veres, P., Burns, E., et al. 2017, ApJL, 848, L14

Gottlieb, O., Nakar, E., \& Piran, T. 2018, MNRAS, 473, 576

Gottlieb, O., Nakar, E., Piran, T., \& Hotokezaka, K. 2017, ArXiv e-prints, arXiv:1710.05896

Granot, J., Gill, R., Guetta, D., \& De Colle, F. 2017, ArXiv e-prints, arXiv:1710.06421

Haggard, D., Nynka, M., Ruan, J. J., et al. 2017, ApJL, 848, L25

Hallinan, G., Corsi, A., Mooley, K. P., et al. 2017, Science, 358,1579

Hotokezaka, K., Kiuchi, K., Kyutoku, K., et al. 2013, PhRvD, 87, 024001

Hotokezaka, K., Kiuchi, K., Shibata, M., Nakar, E., \& Piran, T. 2018, ArXiv e-prints, arXiv:1803.00599

Huang, Y. F., Wu, X. F., Dai, Z. G., Ma, H. T., \& Lu, T. 2004, ApJ, 605, 300
Kasen, D., Metzger, B., Barnes, J., Quataert, E., \& Ramirez-Ruiz, E. 2017, Nature, 551, 80

Kasliwal, M. M., Nakar, E., Singer, L. P., et al. 2017, Science, 358, 1559

Kathirgamaraju, A., Barniol Duran, R., \& Giannios, D. 2018, MNRAS, 473, L121

Kobayashi, S., Piran, T., \& Sari, R. 1999, ApJ, 513, 669

Kumar, P., \& Zhang, B. 2015, PhR, 561, 1

Lamb, G. P., \& Kobayashi, S. 2017a, MNRAS, 472, 4953

—. 2017b, ArXiv e-prints, arXiv:1710.05857

Lazzati, D., López-Cámara, D., Cantiello, M., et al. 2017a, ApJL, 848, L6

Lazzati, D., Perna, R., Morsony, B. J., et al. 2017b, ArXiv e-prints, arXiv:1712.03237

Levan, A. J., Lyman, J. D., Tanvir, N. R., et al. 2017, ApJL, 848, L28

Lyman, J. D., Lamb, G. P., Levan, A. J., et al. 2018, ArXiv e-prints, arXiv:1801.02669

Margutti, R., Genet, F., Granot, J., et al. 2010, MNRAS, 402,46

Margutti, R., Berger, E., Fong, W., et al. 2017, ApJL, 848, L20

Margutti, R., Alexander, K. D., Xie, X., et al. 2018, ApJL, 856, L18

Mészáros, P. 2006, Reports on Progress in Physics, 69, 2259

Metzger, B. D. 2017, Living Reviews in Relativity, 20, 3

Mizuta, A., Nagataki, S., \& Aoi, J. 2011, ApJ, 732, 26

Mooley, K. P., Nakar, E., Hotokezaka, K., et al. 2018, Nature, 554, 207

Nakar, E. 2007, PhR, 442, 166

Nakar, E., Gottlieb, O., Piran, T., Kasliwal, M. M., \& Hallinan, G. 2018, ArXiv e-prints, arXiv:1803.07595

Nakar, E., \& Piran, T. 2017, ApJ, 834, 28

—. 2018, ArXiv e-prints, arXiv:1801.09712

Nakar, E., Piran, T., \& Granot, J. 2002, ApJ, 579, 699

Nicholl, M., Berger, E., Kasen, D., et al. 2017, ApJL, 848, L18

Pian, E., D'Avanzo, P., Benetti, S., et al. 2017, Nature, 551,67

Piran, T. 1999, PhR, 314, 575

Piro, A. L., \& Kollmeier, J. A. 2018, ApJ, 855, 103

Rees, M. J., \& Mészáros, P. 1998, ApJL, 496, L1

Ruan, J. J., Nynka, M., Haggard, D., Kalogera, V., \& Evans, P. 2018, ApJL, 853, L4

Sari, R., \& Mészáros, P. 2000, ApJL, 535, L33

Sari, R., Piran, T., \& Narayan, R. 1998, ApJL, 497, L17

Savchenko, V., Ferrigno, C., Kuulkers, E., et al. 2017, ApJL, 848, L15 
Shibata, M., Fujibayashi, S., Hotokezaka, K., et al. 2017, PhRvD, 96, 123012

Smartt, S. J., Chen, T.-W., Jerkstrand, A., et al. 2017, Nature, 551, 75

Soares-Santos, M., Holz, D. E., Annis, J., et al. 2017, ApJL, 848, L16

Tanvir, N. R., Levan, A. J., González-Fernández, C., et al. 2017, ApJL, 848, L27

Troja, E., Piro, L., van Eerten, H., et al. 2017, Nature, 551, 71

Troja, E., Piro, L., Ryan, G., et al. 2018, MNRAS, arXiv:1801.06516
Valenti, S., David, Sand, J., et al. 2017, ApJL, 848, L24 van Eerten, H., van der Horst, A., \& MacFadyen, A. 2012, ApJ, 749, 44

van Eerten, H., Zhang, W., \& MacFadyen, A. 2010, ApJ, 722, 235

Villar, V. A., Guillochon, J., Berger, E., et al. 2017, ApJL, 851, L21

Zhang, B., Dai, X., Lloyd-Ronning, N. M., \& Mészáros, P. 2004, ApJL, 601, L119

Zhang, B., Fan, Y. Z., Dyks, J., et al. 2006, ApJ, 642, 354

Zhang, W., \& MacFadyen, A. 2009, ApJ, 698, 1261

Zrake, J., \& MacFadyen, A. I. 2013, ApJL, 769, L29 